\title{
A Power Grand Composite Curves Approach for Analysis and Adaptive Operation of Renewable Energy Smart Grids
}

Damian Giaouris $^{1}$; Athanasios I. Papadopoulos ${ }^{1 *}$;Spyros Voutetakis ${ }^{1}$; Simira Papadopoulou ${ }^{1,2}$;

$$
\text { Panos Seferlis }{ }^{1,3}
$$

${ }^{1}$ Chemical Process Engineering and Energy Resources Institute,

Centre for Research and Technology Hellas, Thermi-Thessaloniki, Greece

${ }^{2}$ Department of Automation Engineering, Alexander Technological Educational Institute of Thessaloniki, Thessaloniki, Greece

${ }^{3}$ Aristotle University of Thessaloniki, Department of Mechanical Engineering,

Thessaloniki, Greece

* Corresponding author: spapadopoulos@cperi.certh.gr 


\begin{abstract}
:
This work proposes the use of the power grand composite curves (PGCC) method to identify energy recovery targets in renewable energy smart-grids and to adaptively adjust their operation in short-term energy requirements through appropriately selected power management strategies (PMS). A PMS is the sequence of decisions offering efficient utilization of resources and equipment to meet specific targets. The aim is to identify the appropriate PMS within recurrent subsequent time intervals that efficiently serves the desired operating goals in view of operating variability. This is approached by predicting the PGCC for a time horizon extending into the future. Subsequently, the PGCC is appropriately shifted to set a target for the minimum energy inventory needed by the end of the current interval for which decisions about the system operation are sought in order to satisfy the system operating goals. The target energy inventory is guaranteed in the current interval by selecting the PMS that best matches the identified target. A formal mathematical framework is presented, associating pinch analysis with PMS within a generic model considering numerous structural and temporal grid interactions. The proposed method is implemented on an actual hybrid smart grid considering multiple RES-based energy generation and storage options.
\end{abstract}

Keywords: Smart grids, Energy Management, Power Pinch Analysis, Grand Composite Curves 


\section{Nomenclature}

AEEND Available excess electricity for next day.

BAT Battery.

$B F \quad$ Low pressurized cylinders.

CMP Compressor.

DSL Diesel generator.

EL Electrolyzer.

$F_{n}^{I n} \quad$ Input flow at node $n$.

$F_{m \rightarrow n}^{\text {Out }} \quad$ Output flow from node $m$ to node $n$.

FC Fuel cell.

FT Long-term storage tank.

$H \quad$ Overall time span.

H2HP Hydrogen in high pressure.

$H 2 L P \quad$ Hydrogen in low pressure.

$\mathrm{H} 2 \mathrm{O}$ Water.

HRES Hybrid renewable energy system.

LD Load.

Lo Lower desired limit.

MOES Maximum outsourced energy supply.

min Minimum value of SOAcc ${ }^{l}$.

$\max \quad$ Maximum value of SOACC ${ }^{l}$.

OES Outsourced energy supply.

PGCC Power grant composite curve.

PMS Power Management Strategies.

POW Electrical power.

$P V \quad$ photovoltaic panels

$Q \quad$ Set of all available PMS.

RES Renewable Energy Sources.

$S F_{n} \quad$ External Input at node $n$.

SOAcc ${ }^{l} \quad$ State of accumulator $l$.

$S O A c c_{\text {min }}^{l} \quad$ Initial value of $S O A c c^{l}$ that produces the minimum value of $S O A c c^{l}$. 


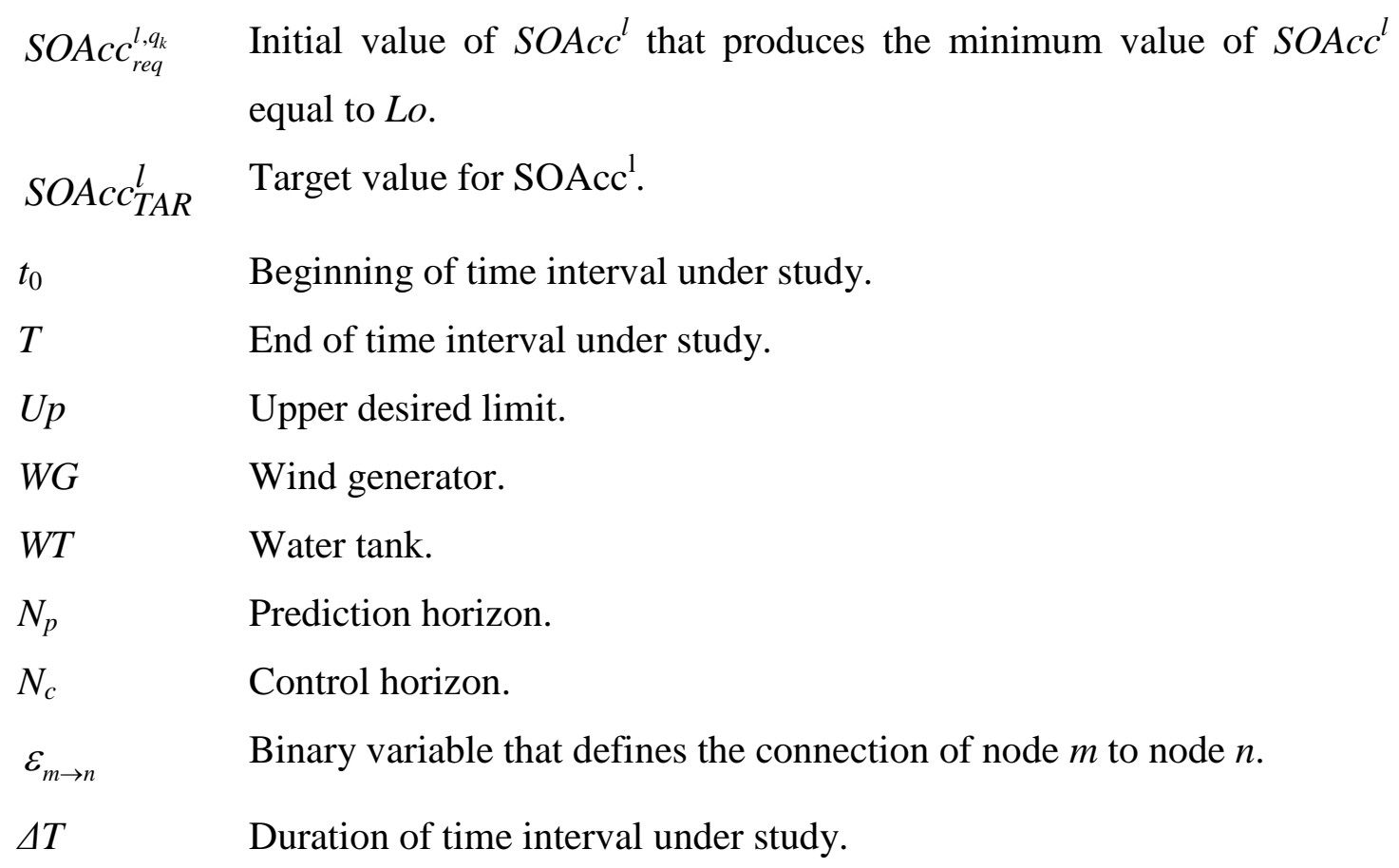

\section{Introduction}

Hybrid power generation systems transform RES into dependable power flows by simultaneous utilisation of different types of energy conversion and storage equipment (e.g. photovoltaic panels, wind generators, fuel cells, electrolysers for hydrogen production, accumulators and so forth) to address the intermittent nature of largely unpredictable environmental phenomena. The resulting infrastructures combine multiple subsystems into a power grid that needs to operate efficiently, while satisfying power demands with minimum participation of non-RES components. This is generally approached through the implementation of operating patterns and control schemes, often coined as PMS [1]. PMS typically account for decisions regarding the appropriate instant to activate/deactivate different energy conversion subsystems, the duration and characteristics (e.g., level of intensity) of such operation, the amount/type of energy carrier to use (e.g., electric power or hydrogen) and so forth. The involvement in grids of numerous subsystems of heterogeneous technical features results in a large number of potential PMS that allow feasible operation. Efficient operation requires the selection and implementation of multiple PMS for periods of time 
according to the grid structure and state. This is a non-trivial task that necessitates intense effort to review, analyse and eventually exploit the complex interactions observed among the subsystems. Process integration methods in the form of the recently developed power pinch concepts (grand composite curves [2] and composite curves [3]) may be used to considerably facilitate the analysis of hybrid power generation grids. Such methods have been inspired from the well-known heat pinch [4] and evolved to sophisticated tools [5] that allow for the analysis of complex energy systems based on the identification of insights pointing towards promising design and operating decisions [6]. A major advantage of these methods is their implementation in the form of intuitive and easy to develop graphical interfaces (e.g., grand composite curves), whereas the underlying principles are often efficiently represented using rigorous mathematical tools (e.g., flexible process models combined with optimization algorithms). Regardless of the realization, pinch methods allow the user to easily identify, review and analyse potentially useful design and operating options. A very recent overview of pinch analysis and mathematical programming for process integration is presented in [7]. Focusing on electrical systems, pinch-based analysis methods utilize composite or grand composite curves similarly to the traditional heat pinch however the associated sink and source streams are plotted in power versus time diagrams. In this context, a method proposing the identification of energy recovery targets using the grand composite curves (GCC) analysis approach was reported in [2] addressing the optimal sizing of power generation systems in the form of an optimization problem. Work presented in [3] proposed the power pinch analysis (PoPA) method to determine the minimum electricity targets for systems comprising hybrid renewable energy sources. The graphical power pinch analysis method takes the form of numerical tools in [8] such as the power cascade analysis (PoCA) and storage cascade table (SCT) in order to facilitate the precise allocation of power and electricity targets in power generation systems. Work presented in $[9,10]$ extends the numerical power pinch method by additionally considering power losses during conversion, transferring and storage. The method is 
applied in the optimization of a pumped hydro-storage system in [11], while it is also extended in [12] to address the optimal sizing of hybrid power generation systems. Recent work presented in [13] proposes the outsourced and storage electricity curves (OSEC) to visualise the required minimum outsourced electricity and the current storage capacity at each time interval during startup and operation of hybrid power systems. Heuristics for load shifting that may lead to further reductions of the maximum storage capacity and the maximum power demand in hybrid systems are also proposed. Work presented in [14] proposes the stand-alone hybrid system power pinch analysis method (SAHPPA), which is a graphical tool employing new ways of utilising the demand and supply composite curve methods. Work presented in $[15,16]$ proposes an MILPbased transhipment model for targeting the outsourced electricity requirements and allocating renewable electricity and storage components to demands in hybrid power systems. Recently, work presented in [17] adapted the power pinch concept in the Electricity System Cascade Analysis (ESCA) approach to optimize distributed energy generation systems, while this approach was used for the optimum sizing and operation of a solar/wind/battery hybrid system [18].

The energy recovery targets identified by pinch-based methods are represented as composite or grand composite curve graphs; their shape reflects on grid operating conditions which may be achieved by different equipment utilization sequences and operating patterns, updated within and between different instants and implemented as PMS in hybrid renewable energy grids. Without considering pinch-based analysis methods, previous works have addressed the design and operation of hybrid energy systems using empirically specified PMS for DC distribution in buildings [19], PV/Wind/Fuel cell systems [20], stand-alone systems with hydrogen support [21], hybrid vehicles [22], hybrid systems with renewables and hydrogen [23-25] and micro-grids with micro-turbines, fuel cells and batteries [26]. PMS incorporated in optimization have also been considered in Combined Heat and Power (CHP) systems [27], distributed energy systems [28], industrial CHP systems [29], polygeneration micro-grids [30], internally reconfigurable networks 
[31], trigeneration systems [32], micro combined heat and power systems [33] and stand-alone hybrid systems [34]. Finally, PMS in optimal control methods have also been considered for hybrid vehicles [35], microgrids [36], stand-alone systems [37] and renewable energy and hydrogen systems [38]. Regardless of the employed method, a key requirement for the development of efficient PMS is the existence of an inclusive underlying model able to capture all possible structural and temporal interactions among the grid subsystems. Such a model is adopted in this work from [1] where it was recently proposed by the authors. It employs generic flowsheet modelling concepts to describe flows and tasks of conversion and accumulation, integrating streams utilized in multi-component material/energy conversions and resulting in flexible representations. These are coupled with the use of logical propositions assembled around generic temporal operating features, supporting the development of conditional statements that represent operating requirements as constraints. Although the proposed model allows the development of numerous PMS, the efficient system operation depends on the way that the derived PMS is utilized. Conventionally, hybrid power generation systems mainly utilize a PMS selected through simulation or optimization-based methods and repeated throughout the cyclic system operation. This is clearly inefficient for two reasons. First, the PMS selected for the entire system operating time span may exhibit an efficient exploitation of the available resources in several time intervals against other candidate PMS. However, it usually results in inefficient operation in several others leading to energy waste and increased non-RES or outsourced power utilization. Furthermore, the long-term use of a rigid PMS results in inefficient operation in case of previously unaccounted variability such as unexpected weather or demand fluctuations.

To address the above challenges the present work exploits the power pinch analysis methods to develop a more effective approach involving the repeated identification and implementation of a new PMS for the immediate short term future horizon. This results in a set of efficient PMS specifically tailored to the power demand/supply requirements imposed by the expected variability 
conditions (e.g., available weather profiles and so forth). It also allows the system to react and efficiently adapt to unexpected variability, hence upgrading conventional grids with smart capabilities. Smart grid architectures have been previously proposed in published literature for hybrid power generation systems addressing the coupling of microgrids [39], considering various configurations [40], utilizing multi-agent methods [41] and well known control methods to address economic performance [42] and real-time operation [43]. The smart capabilities proposed in this work exploit the power pinch methods and take the form of a supervisory management layer acting on top of the available PMS and performing two functions: a) the identification of RES-based energy recovery targets within a short term future horizon where changes in exogenous conditions are anticipated and can be predicted, and b) the reallocation of the grid subsystems based on the PMS that best match the targets identified within the considered horizon. The benefits of the recently developed power pinch analysis methods $[2,3]$ are employed in support of the first function. The second function is implemented through the PMS selection from an inclusive pool of potential alternatives based on systemic and flexible models. Pinch analysis is associated with multiple different ways of operating a smart grid through various PMS, while ways of addressing cases with multiple pinches and operating goals are proposed. Pinch analysis is also formally associated with a generic model considering numerous potential structural and temporal interactions observed in smart grids and transforming them into PMS. An algorithmic sequence is also proposed that enables the automated implementation of the method for yearround system operation. The proposed developments are used for the efficient operation of a power generation microgrid that also involves hydrogen production, storage, and utilization considering multiple PMS as decision options. The considered microgrid is an actual system currently in operation in Xanthi, Greece [21], constructed and commissioned by the authors. The rest of the paper is organized as follows: Section 2 presents an overview of the microgrid that motivated this work, the observed operating challenges and the main modelling concepts. Section 
3 presents a detailed analysis regarding the implementation of power pinch in smart grids and the proposed adaptive management method. Section 4 presents implementation details of a case study and a discussion of the obtained results, while section 5 presents some concluding remarks.

\section{System and models}

\subsection{Motivating system and associated challenges}

Prior to presenting the proposed management approach it is necessary to describe the isolated hybrid RES-based power system that originally motivated this work. It consists of photovoltaic panels $(P V)$ and wind generators $(W G)$ for power generation from RES. Surplus energy is supplied to a water electrolyzer $(E L)$ for hydrogen production after the specified load demand $(L D)$ for a targeted application is satisfied. The produced hydrogen is temporarily stored in low pressurized cylinders $(B F)$, and then through a hydrogen compressor $(C M P)$ in long-term storage tanks $(F T)$. In cases of energy deficit, this hydrogen is utilized by a fuel cell $(F C)$ to provide the needed power to the system. Lead-acid accumulators $(B A T)$ are used to regulate the power flows in the system through frequent charging and discharging cycles induced by the RES variability. A diesel generator $(D S L)$ is also included to the system and utilized only in cases of emergency when power demands of the application cannot be covered by RES or stored hydrogen, whereas a water tank (WT) is installed to support the $E L$ and $F C$ operation. Obviously, suitable power converters ensure the efficient regulation of electricity. The models used in this work were previously presented and experimentally validated by the authors in [23-25]. Fig. 1, shows the aforementioned isolated microgrid. 


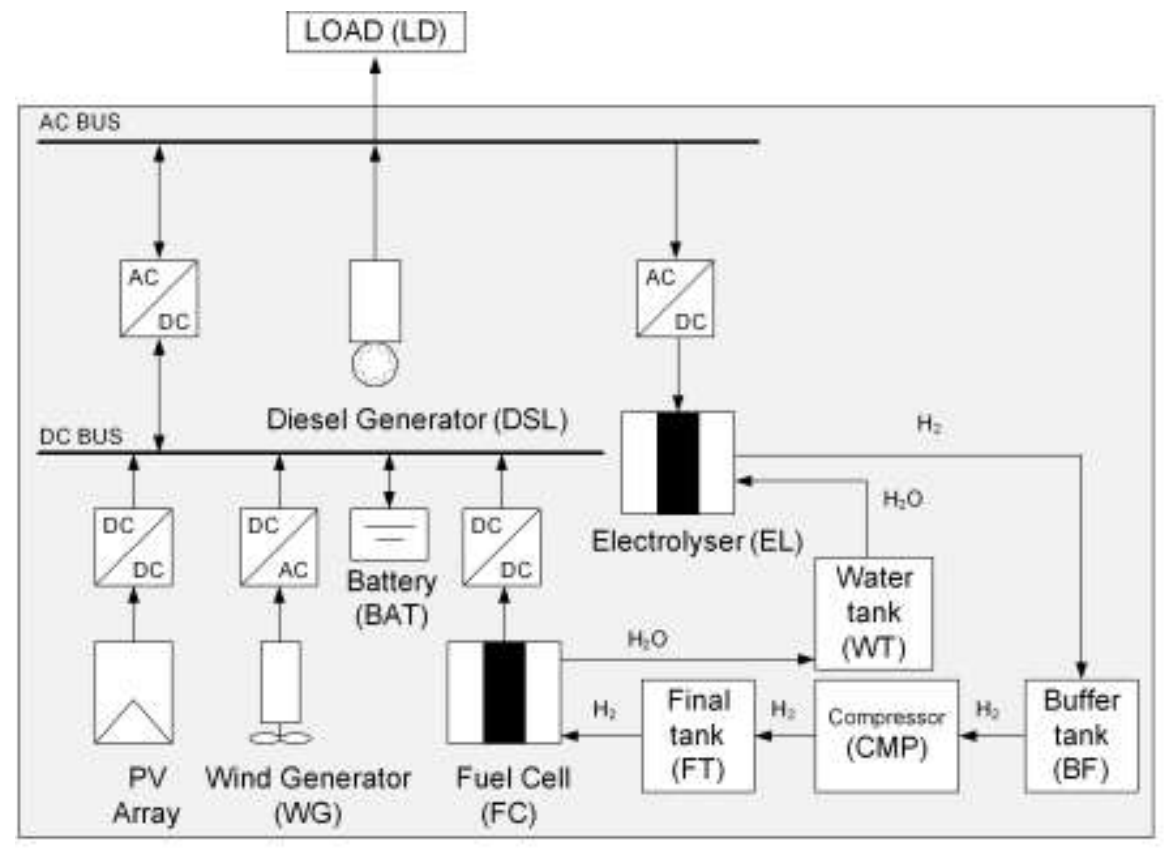

Figure 1: Isolated microgrid used as a case study [1].

The key features of this system are flows and accumulation of both materials and energy at different time instants. The instant and duration of activation or deactivation of each unit are important operating parameters as they determine the frequency of activation for each device. The operating pattern duration together with the frequency affect the replacement and maintenance schedule of sensitive equipment like $E L, F C$, and $B A T$. The capacity of different types of accumulators is also important because it directly affects the activation frequency. Excess power or hydrogen needs to be stored and used at a later instance, while deficits need to be served by appropriate accumulators based on the type and the conditions of the required energy carrier. All these features add significant complexity in the decision making around a system that is driven by variable and difficult to predict weather conditions. Pinch-based analysis methods provide clear insights into these interactions by treating all sub-systems as demand and supply nodes over time.

\subsection{Structural and temporal modelling}

The characteristics of the motivating system may be generalized to facilitate the analysis using pinch-based methods. Regardless of equipment and operating conditions, the system flowsheet 
represents a structure containing sub-systems interconnected through streams, facilitating the power supply of an end-user. Based on this observation all the particular system features can be organized under the following generic sets:

a) The set of States $(S t)$ representing the composition of streams interconnecting different subsystems and consisting of two main subsets including the states of Energy (Nrg) and Matter (Mat), hence $S t=\left\{S t^{N r g}, S t^{M a t}\right\}$.

b) The set of Resources $(R s)$ indicating the type of equipment employed to perform conversion and accumulation tasks and including the subsets of accumulators $R s^{A c c}$ and converters $R s^{C o n v}$, hence $R s=\left\{R s^{A c c}, R s^{C o n v}\right\}$.

Focusing these concepts on the particular features considered in this system without any loss of generality, the set of states may be directly defined as $S t=\{P O W, H 2 H P, H 2 L P, H 2 O\}$, with $P O W \in S t^{N r g}$ and $\{H 2 H P, H 2 L P, H 2 O\} \in S t^{M a t}$ where POW is electrical power (stored in BAT), $H 2 H P$ is hydrogen in high pressure (stored in FT), H2LP is hydrogen in low pressure (stored in $B F$ ) and $\mathrm{H} 2 \mathrm{O}$ is water (stored in $W T$ ). Since the investigated systems incorporates particular types of equipment to perform specific conversion and accumulation tasks the employed equipment may be directly organized into the sets of accumulators $R s^{A c c}=\{B A T, F T, B F, W T\}$ and converters $R S^{C o n v}=\{P V, W G, D S L, E L, F C, C M P, L D\}$.

\section{Flows and accumulation}

Based on these sets the flows of energy or materials may be calculated as follows:

$$
F_{n}^{I n, j}(t)=S F_{n}^{j}(t)+\sum_{l}\left(\varepsilon_{l \rightarrow n}(t) F_{l \rightarrow n}^{O u t, j}(t)\right)+\sum_{k}\left(\varepsilon_{k \rightarrow n}(t) \cdot F_{k \rightarrow n}^{O u t, j}(t)\right)
$$

where $l \in R s^{A c c}, k \in R s^{C o n v}, n \in R s, j \in S t, F_{l \rightarrow n}^{O u t, j}(t)$ is the output of accumulator $l$ that goes to device $n$ in state $j, F_{k \rightarrow n}^{O u t, j}(t)$ is the flow of converter $k$ that goes to device $n$ in state $j, S F_{n}^{j}(t)$ is a 
possible external signal or flow (e.g. solar radiation), $\varepsilon_{l \rightarrow n}(t)$ is a binary variable that becomes 1 when accumulator $l$ feeds device $n$ in state $j$ and $\varepsilon_{k \rightarrow n}(t)$ is a binary variable that becomes 1 when converter $k$ feeds device $n$ in state $j$.

The accumulators are characterised by the amount of stored energy or material which can generally be defined as the normalised amount of the stored state:

$\operatorname{SOAcc}^{l}(t)=\operatorname{SOAcc}^{l}\left(t^{-}\right)+\frac{F_{k \rightarrow l}^{\text {in,j }}(t)-F_{l \rightarrow k}^{\text {out }, j}(t)}{C_{l}}$

where $\operatorname{SOACC}^{l}(t)$ is the amount of stored state $j$ in accumulator $l$ at time instant $t$, the symbol $t^{-}$ is used to account for the previous observation instant and $C_{l}$ is the capacity of accumulator $l$ in state $j$. Considering that the investigated accumulators may store only one state, symbol $j$ is omitted for simplicity from terms SOAcc and $C$.

\section{Activation and hysteresis band}

To apply the above balance equations it is necessary to define binary variables $\varepsilon$ of equation (1) as they enable the activation/deactivation of connections between devices $m$ and $n$ based on temporally evolving constraints. Decisions whether to activate a connection are based on conditions considering: a) the availability of material or energy from device $m \in R s$, b) the requirement for material or energy of device $n \in R s, n \neq m$, and c) additional specific conditions that are not associated with the above two and may be desirable. In this context, variable $\varepsilon_{m \rightarrow n}(t)$ can be defined in the form of a generic logical proposition as follows:

$$
\begin{aligned}
& \varepsilon_{m \rightarrow n}(t)=L\left(\varepsilon_{m \rightarrow n}^{A v l}(t), \varepsilon_{m \rightarrow n}^{R e q}(t), \varepsilon_{m \rightarrow n}^{G e n}(t)\right) \\
& \varepsilon_{m \rightarrow n}^{i}(t)=L_{m \rightarrow n}^{i}\left(L_{m \rightarrow n}^{\text {SOAcc }}\left(\rho_{m \rightarrow n}^{\text {SOAccl }}(t), r_{m \rightarrow n}^{\text {SOAccl }}(t)\right)\right) \forall i=\{\text { Avl, Req,Gen }\}, n \in R s^{A c c}, m \in R s
\end{aligned}
$$

In equations (3) and (4) $L$ is a logical operator, indices $A v l$ and $R e q$ correspond to the previously discussed conditions (a) and (b) with respect to variables $\varepsilon$ while index Gen corresponds to 
condition (c) and represents a free use of variable $\varepsilon^{G e n}$ to incorporate any additional desired condition. Binary variables $\rho$ and $r$ are parameters associated with temporal conditions imposed on the power or materials stored in the accumulator $l\left(S O A c c^{l}\right)$. The operation of converters is associated with activation and deactivation (e.g., the fuel cell can take the states ON or OFF) while the accumulators are practically always active $(\mathrm{ON})$. This switching results in the generation of hysteresis zones $[1,44]$, where the converter operation depends on whether it was activated or deactivated in the previous instant. The potential actions involve the following options: a) If the accumulated energy/material $\left(S O A c c^{l}\right)$ value is higher than a pre-specified limit $U p_{m \rightarrow n}^{S O A c c^{l}}(t)$, the converter is not connected to any other device hence it remains idle, b) If it is lower than $\operatorname{Lo}_{m \rightarrow n}^{S O A c c^{l}}(t)$ the converter is connected to another device hence it is activated, c) If it is in the interval between the two limits, it will remain idle if the converter was idle in the previous instant $\left(t^{-}\right)$, or active if it was previously active. In case where a converter is activated when the SOAcc gets higher than a specific value of $S O A c c^{l}$ then the above analysis is reversed. In this context, the range of the hysteresis zone is determined by the difference $U p_{m \rightarrow n}^{\text {SOAcc }}{ }^{l}(t)-L o_{m \rightarrow n}^{\text {SOAcc }}{ }^{l}(t)$. These if-else statements can be represented through generic logical propositions. Each converter is associated with multiple accumulators by which it is affected and activated/deactivated based on their synergy. The SOACC of each accumulator $n$ is divided into 3 zones based on the actions applied on the converter $m$ which is affected. The resulting generic condition has the following form:

$$
\begin{aligned}
\rho_{m \rightarrow n}^{\operatorname{SOAcc}^{l}}(t)= & {\left[\operatorname{SOAcc}^{l}(t)<\operatorname{Lo}_{m \rightarrow n}^{\operatorname{SOAcc}^{l}}(t)\right] \vee } \\
& {\left[\left[\operatorname{Lo}_{m \rightarrow n}^{\operatorname{SOAccl}^{l}}(t)<\operatorname{SOAcc}^{l}(t)<U p_{m \rightarrow n}^{\operatorname{SOAcc}^{l}}(t)\right] \wedge\left[\varepsilon_{m \rightarrow n}\left(t^{-}\right)=1\right]\right] }
\end{aligned}
$$

In equation (5) the first inequality represents the simple ON-OFF behaviour, while the subsequent expression represents the hysteresis behaviour. The recurring implementation of equations (3)-(5) for a desired time horizon results in a PMS. Different PMS result from different combinations of 
logical operators or values imposed on the upper and lower operating limits. Parameter $r$ appearing in equation (4) is used in combination with $\rho$ to impose or ignore a condition if necessary. Clearly, equation (5) shows that there is only a need to investigate three accumulation areas associated with each accumulator $n$. This is simply repeated for every connection. Apparently, the values of the limiting parameters may become additional decision variables for the investigation of the overall system performance. Furthermore, in addition to changing the values of the limiting parameters, different terms can be added, removed or altered in equations (3) to (5) capturing all the potential or desirable connections among different devices.

\section{Proposed method}

\subsection{PGCC for system analysis and targeting}

Let a hybrid renewable energy and storage grid system operating within an overall time span $H$ divided into $k$ equal time intervals that are used for operation related decision making. Each interval is further divided into subintervals $\left[t_{0}, T\right]$ of duration $\Delta T=T$ - $t_{0}$ that are used for simulation hence for the $k^{\text {th }}$ interval $t_{0}=(k-1) \Delta T$ and $T=k \Delta T$. For example, $H$ can be a year that is divided into 365 days, therefore $T=24 \mathrm{~h}$ and $k \in[1,365]$ for which a set of operating decisions are determined. Each day is divided into intervals of $1 \mathrm{~h}$ for the simulation of the system behaviour. Also, assume a set $Q$ of various PMS that can be used in a hybrid system, for example $Q=\left\{P M S_{i}\right\}, i \in \mathbb{N}$ as given in [1]; a different PMS can be employed in each interval $k$, i.e. $Q=\left\{P M S_{i, k}\right\}, i \in \mathbb{N}, k \in[1,365]$ In order to simplify the notation we denote these PMS with lower case letters, hence $Q=\left\{q_{k}, p_{k}\right.$, $\left.h_{k} \ldots\right\}$. Essentially each PMS is a potential operating combination or group of combinations resulting from equations (3)-(5) that define(s) a function $f$ such that $P M S_{i, k}=f\left(\varepsilon^{q_{k}}, S O A c c^{l, q_{k}}(t), k\right)$. Notice that the variables $\varepsilon$ and $S O A c c^{l}$ have now another superscript to denote that the $q^{\text {th }}$ PMS is used in interval $k$. A major requirement in systems like the one under study is to eliminate the need for external supply (of $N R G$ or MAT) when the 
$\operatorname{SOAcc}^{l, q_{k}}(t)$ for any accumulator drops below a specific value. For example, if $l=B A T$ then extra electrical power should be retrieved from either a $D S L$ or another microgrid. Similarly, if $l=F T$ hydrogen may be required from either an external supplier or the DSL that will support the operation of the $E L$.

In this context, the PGCC (or graph of $S O A c c^{l}$ ) corresponding to a PMS $q_{k}$, for three initial values of $S O A c c^{l}$, around an accumulator $l$ and a given realization of exogenous weather conditions is shown in Fig. 2a. Obviously, for each initial value of $S O A c c^{l}$ different responses are obtained that generally maintain the same qualitative characteristics. In case that the SOAcc ${ }^{l}$ drops below a specific limit the system requires external or outsourced energy supply (OES). Following the notation of the previous section the limit $L o \equiv \operatorname{SOAcc}^{l, q_{k}}\left(t_{L o}\right)$ corresponds to a value where extra supply will be required in order to increase $\operatorname{SOAcc}^{l, q_{k}}(t)$. For example if $l=B A T$, then this value is the discharge limit below which the energy required to keep satisfying the $L D$ will have to be introduced from an external source (e.g., from a non-RES system such as the DSL). In order to avoid unnecessary complexity and to mathematically define the OES, Fig. 2b shows only one PGCC and the limit $\min \equiv \operatorname{SOACc}^{l, q_{k}}\left(t_{\min }\right)$ corresponding to a value indicating the minimum $\operatorname{SOACc}^{l, q_{k}}(t)$ that was reached for the given PMS starting from $\operatorname{SOACc}^{l, q_{k}}\left(t_{0}\right)$. It has to be noted here that $t_{L o}$ and $t_{\min }$ depend on the PMS $q_{k}$ that is employed and on the value of $\operatorname{SOAcc^{l,q_{k}}}\left(t_{0}\right)$. However, to avoid introducing additional complexity in the notation this is not mentioned in most equations unless it is necessary. 


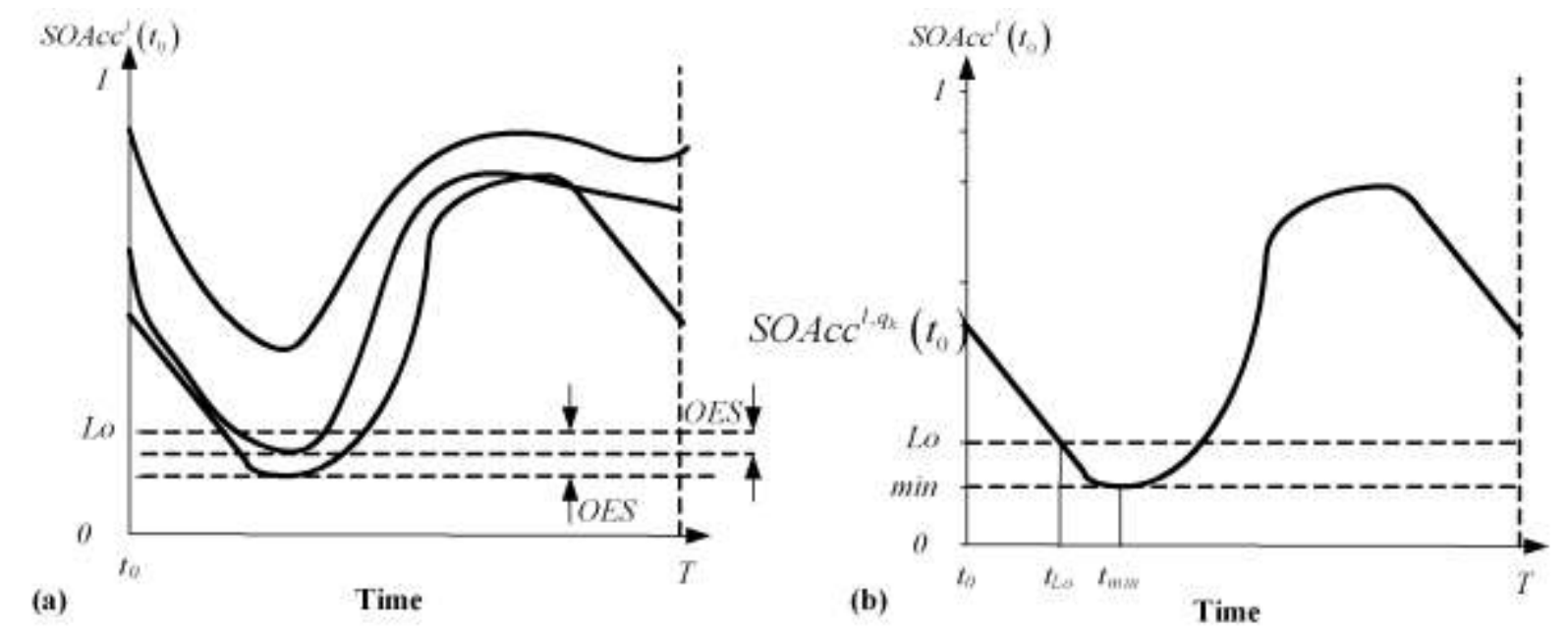

Figure 2: PGCCs for various initial conditions of $S O A c c$; a) shows the response of the SOAcc for three different initial values and b) shows the response with the lowest value of SOAcc.

The OES for each $\operatorname{SOAcc}^{l, q_{k}}\left(t_{0}\right)$ is therefore defined as:

$\operatorname{OES}^{l, q_{k}}(k)=\operatorname{SOAcc}^{l, q_{k}}\left(t_{L O}\right)-\operatorname{SOAcc}^{l, q_{k}}\left(t_{\text {min }}\right)$

The available energy at the end of the time horizon (AEEND) is defined as:

$\operatorname{AEEND}^{l, q_{k}}(k)=\operatorname{SOAcc}^{l, q_{k}}(T)-\operatorname{SOAcc}^{l, q_{k}}\left(t_{L O}\right)$

The initial value $\operatorname{SOAcc}^{l, q_{k}}\left(t_{0}\right)$ that will produce the minimum $\operatorname{SOAcc}^{l, q_{k}}\left(t_{\text {min }}\right)$ is denoted as $S O A c c_{\min }^{l, q_{k}}\left(t_{0}\right)$ and the maximum outsourced energy supply (MOES) [3] is defined as:

$\operatorname{MOES}^{l, q_{k}}(k)=\operatorname{SOAcc}^{l, q_{k}}\left(t_{L O}\right)-\operatorname{SOAcc}^{l, q_{k}}\left(t_{\text {min }}\right): \operatorname{SOAcc}^{l, q_{k}}\left(t_{0}\right)=\operatorname{SOAcc}_{\min }^{l, q_{k}}\left(t_{0}\right)$

Equation (8a) also takes the form of (8b) which highlights the relation between MOES and OES:

$\operatorname{MOES}^{l, q_{k}}(k)=\max _{\operatorname{SOAcc}^{l, q_{k}}\left(t_{0}\right)}\left\{\operatorname{OES}^{l, q_{k}}(k)\right\}=\max _{\operatorname{SOAcc}^{l, q_{k}}\left(t_{0}\right)}\left\{\operatorname{SOAcc}^{l, q_{k}}\left(t_{L O}\right)-\operatorname{SOAcc}^{l, q_{k}}\left(t_{\text {min }}\right)\right\}$

Based on the pinch analysis principles the PGCC can be shifted (continuous black line in Fig. 3) until it touches the Lo limit i.e. $\operatorname{SOACc}^{l, q_{k}}\left(t_{\text {min }}\right)=L o$. This is the pinch point and indicates the minimum amount of energy at $t=t_{0}$ that is required from internal resources in order to avoid using outsourced electricity resources for the given PMS and SOACc. Basically, this is the most 
constrained point in the system. Therefore the minimum initial $S O A c c^{l, q_{k}}$ that is required in order to eliminate the requirement for external supply is defined as:

$\operatorname{SOACc}_{r e q}^{l, q_{k}}\left(t_{0}\right)=\operatorname{SOACc}_{\min }^{l, q_{k}}\left(t_{0}\right)+\operatorname{MOES}^{l, q_{k}}(k)$

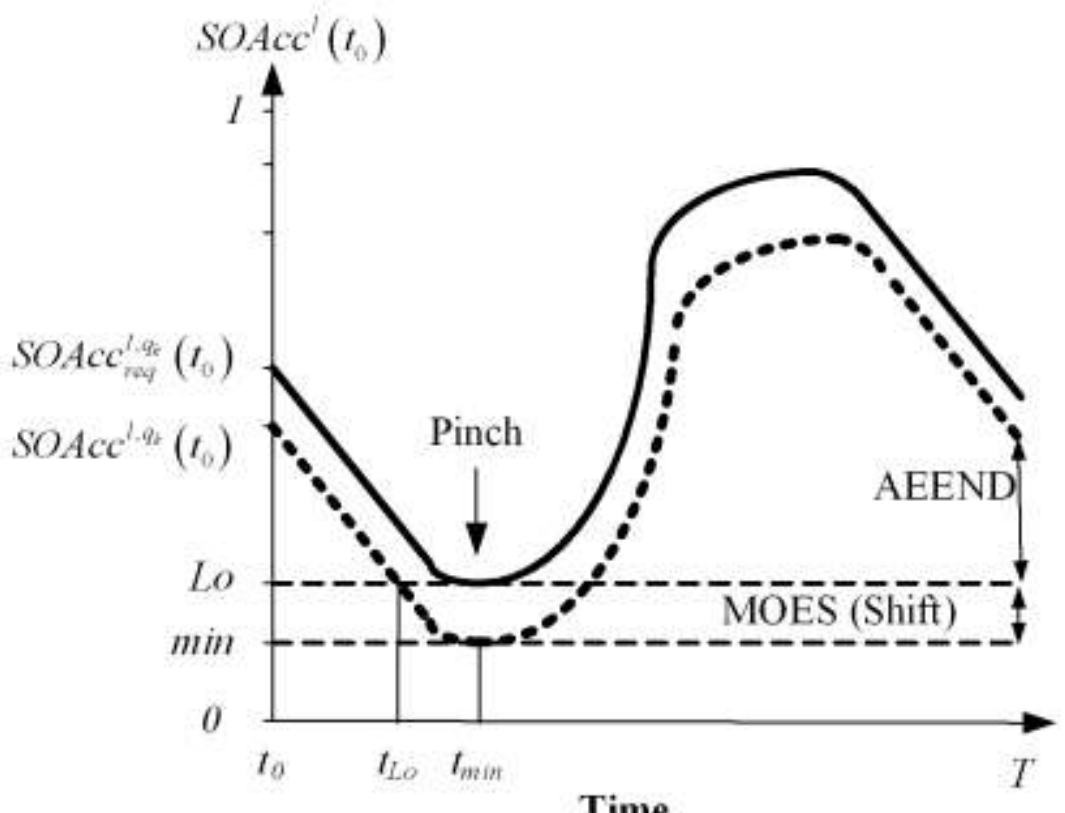

Time

Figure 3: Concept of Pinch Analysis.

The values of $\operatorname{SOACc}^{l, q_{k}}\left(t_{\text {min }}\right)$ and $S O A c c_{\text {min }}^{l, q_{k}}\left(t_{0}\right)$ may be determined visually from Fig. 3. Alternatively, they may be calculated from the following equations:

$\operatorname{SOAcc}^{l, q_{k}}\left(t_{\text {min }}\right)=\min _{t \in\left[t_{0}, T\right]}\left\{\operatorname{SOAcc}^{l, q_{k}}(t)\right\}$

$\operatorname{SOAcc}_{\min }^{l, q_{k}}\left(t_{0}\right)=\operatorname{SOAcc}^{l, q_{k}}\left(t_{0}\right): \operatorname{MOES}^{l, q_{k}}(k)=\operatorname{OES}^{l, q_{k}}(k)$

Note that in this work the term OES (the same also holds for AEEND) refers to energy in order to maintain consistency with previously proposed power pinch analysis terminology [3]. However, it may also be used in a more general context; for example it can be the extra energy required to charge the battery or the amount of hydrogen to fill the final tank. 


\subsection{Effects of the hysteresis zones}

With respect to equations $(10 a, b)$ note that by starting from different initial values of $\operatorname{SOACc}^{B A T, q_{k}}(t)$ it is not generally expected to obtain a new PGCC of the same form as previously (i.e. the values of $S O A c c^{B A T, q_{k}}(t)$ will not be shifted by MOES for every $t$ unless the hysteresis zones (i.e. $U p$ and $L o$ limits) of all the devices are also shifted accordingly. This can be seen in Fig. 4 which presents an example of activating and deactivating a $F C$ based on the state of charge of a $B A T$. The $S O A c c^{B A T, q_{k}}(t)$ starts from $\operatorname{SOACc}_{\text {min }}^{B A T, q_{k}}\left(t_{0}\right)$ and the $F C$ is activated at $t=t_{O N}$ and deactivated at $t=t_{O F F}$ (based on (5)). In Fig. 4a the solid trace corresponding to the shifted PGCC shows the $S O A c c^{B A T, q_{k}}(t)$ when it starts from $\operatorname{SOAcc}_{r e q}^{B A T, q_{k}}\left(t_{0}\right)$ without changing the operating or hysteresis zone of the $F C$. From that figure it can be clearly seen that as the $F C$ is activated after $t=t_{O N}$, the response of the $S O A c c^{B A T, q_{k}}(t)$ starting from $\operatorname{SOAcc}_{r e q}^{B A T, q_{k}}\left(t_{0}\right)$ drops below the limit $L o$ (despite the correctly calculated shift given by equation (8)). On the other hand, if the hysteresis zone of the fuel cell is also changed by $\operatorname{MOES}^{B A T, q_{k}}(k)$ units (see Fig. 4b) then the relation $\operatorname{SOAcc}^{B A T, q_{k}}\left(t_{\text {min }}\right)=\operatorname{SOAcc}^{B A T, q_{k}}\left(t_{L O}\right)$ is obtained. In other words, equations (8a) or (8b) provide the necessary shift that must be imposed on $\operatorname{SOAcc^{BAT,q_{k}}}\left(t_{0}\right)$ and on $\operatorname{Lo}_{F C \rightarrow B A T}^{S O A c^{B A T}}(t), U p_{F C \rightarrow B A T}^{S O A c c^{B A T}}(t)$. This affects equation (5) which determines the activation and deactivation of converters based on the state $(S O A c c)$ of the associated accumulators. Note that shifting the hysteresis zones serves the goal of avoiding $S O A C c^{B A T}$ values below a limit Lo (for example in order to avoid the deep discharging of the battery). If the goal is to avoid the usage of a particular device (e.g., DSL) then the hysteresis zone of that device should not be shifted. 


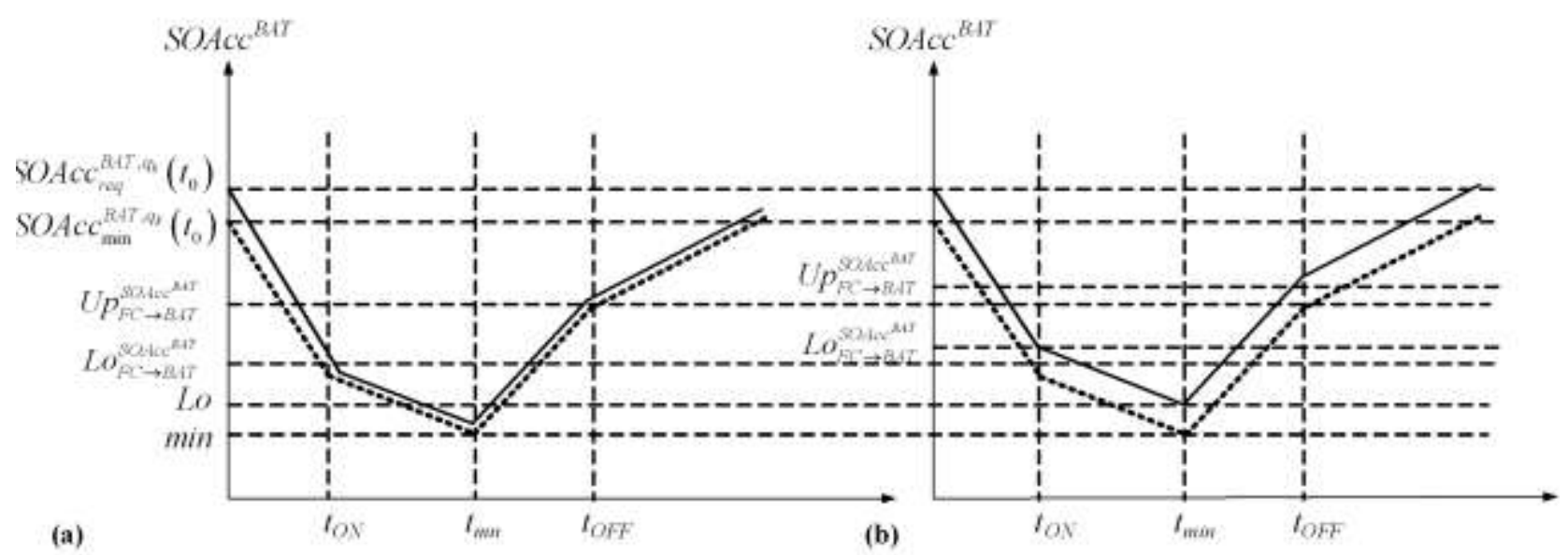

Figure 4: Effect of the hysteresis zone in the shift of the PGCC; a) shifted SOAcc response when the operational limits of the FC have not been shifted, b) response with the shifted operating region of the FC.

Another important effect of the hysteresis zones in the aforementioned shift involves the considered sampling time resolution. In this work the resolution involves hourly time intervals. For this case it has been observed that for two nearby initial $S O A c c^{B A T}$ values the subsequent $S O A C c^{B A T}$ values may deviate as shown in Fig. 5. This means that in some cases even if the imposed shift is intended to avoid the violation of a $L o$ type limit, this may not be possible. This issue depends on the sampling time resolution and becomes negligible as the resolution increases. In the case under study, the range of the deviation is illustrated numerically by the following example. Fig. 5 shows that at $t=1$ the trace with the circles starts at $S O A c c^{B A T}=0.214$ and the one with the solid squares at $S O A c c^{B A T}=0.213$ (i.e. they are both above the limit of 0.2 ). At $t=3$ the trace that started at 0.214 is at 0.2006 while the other is at 0.1996 . Although until $t=3$ the $S O A c c^{B A T}$ values are practically the same, in the second case the $D S L$ is activated at $t=3$ because $S O A c c^{B A T}$ drops below the limit of 0.2. As a result, the $S O A c c^{B A T}$ is increasing from 0.1996 and at the next time instant $S O A C c^{B A T}=0.2004$. In the other case it is decreasing from 0.2006 at $t=3$ to $S O A C c^{B A T}=0.1938$ at the next instant. Hence, by imposing an appropriate shift to start from a higher value of $S O A C c^{B A T}$ does not always imply a higher value of $\operatorname{SOACc}\left(t_{\text {min }}\right)$ (i.e. the pinch 
may still be slightly violated). For a time resolution of the order used in this work, this violation is practically negligible as shown in the example.

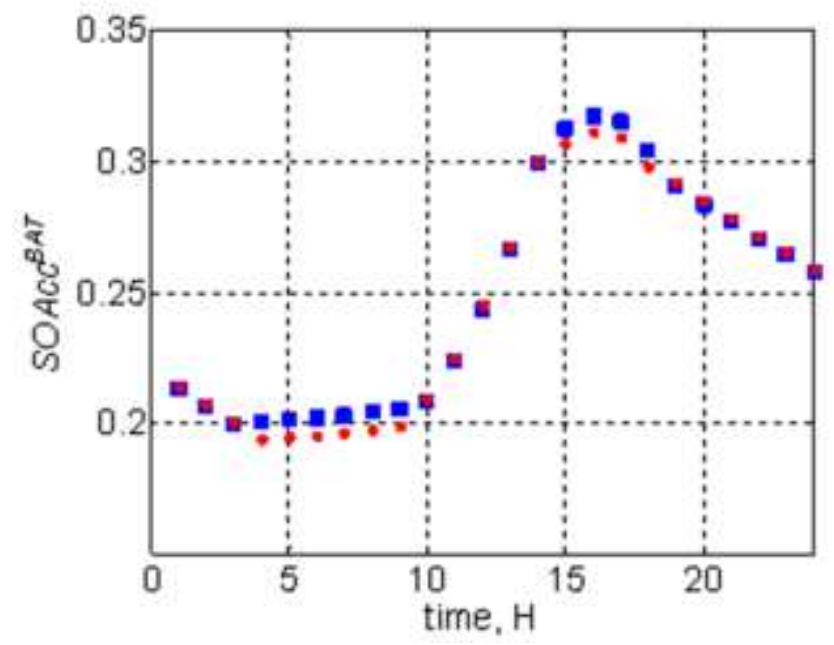

Figure 5: Effect of the hysteresis zones on the shape of the PGCC.

\subsection{Evaluation of multiple goals}

Equation (9) presents an energy target developed for the case when the goal is to completely avoid the use of external or non-RES sources, for a given PMS $q_{k}$. An interesting question here is what happens when there are additional multiple goals or limits to be satisfied. This subsection extends the previous analysis in the implementation of pinch principles to combine various different constraints and find one (if possible) shift that will satisfy all (or most) of them.

\section{Case 1: Multiple Lo limits}

This case may appear if for example in the system under study a restriction is set in the usage of both the DSL and $F C$, which may be represented by limits $L o_{1}$ and $L o_{2}$ in Fig. 6. The resulting PGCC may be shifted to pinch both limits with equation (8) holding in both cases. To choose the pinch that will be implemented, the shift may be expressed as follows:

$$
\operatorname{MOES}^{l, q_{k}}(k)=a_{1} \cdot \operatorname{MOES}_{1}^{l, q_{k}}(k)+a_{2} \cdot \operatorname{MOES}_{2}^{l, q_{k}}(k)
$$

where that weights $a_{1}$ and $a_{2}$ are chosen based on the desired shift; if it is desired to satisfy the limit $L o_{1}$ then $a_{1}=1$ and $a_{2}=0$, while the opposite will hold if the desired limit is the $L o_{2}$. 

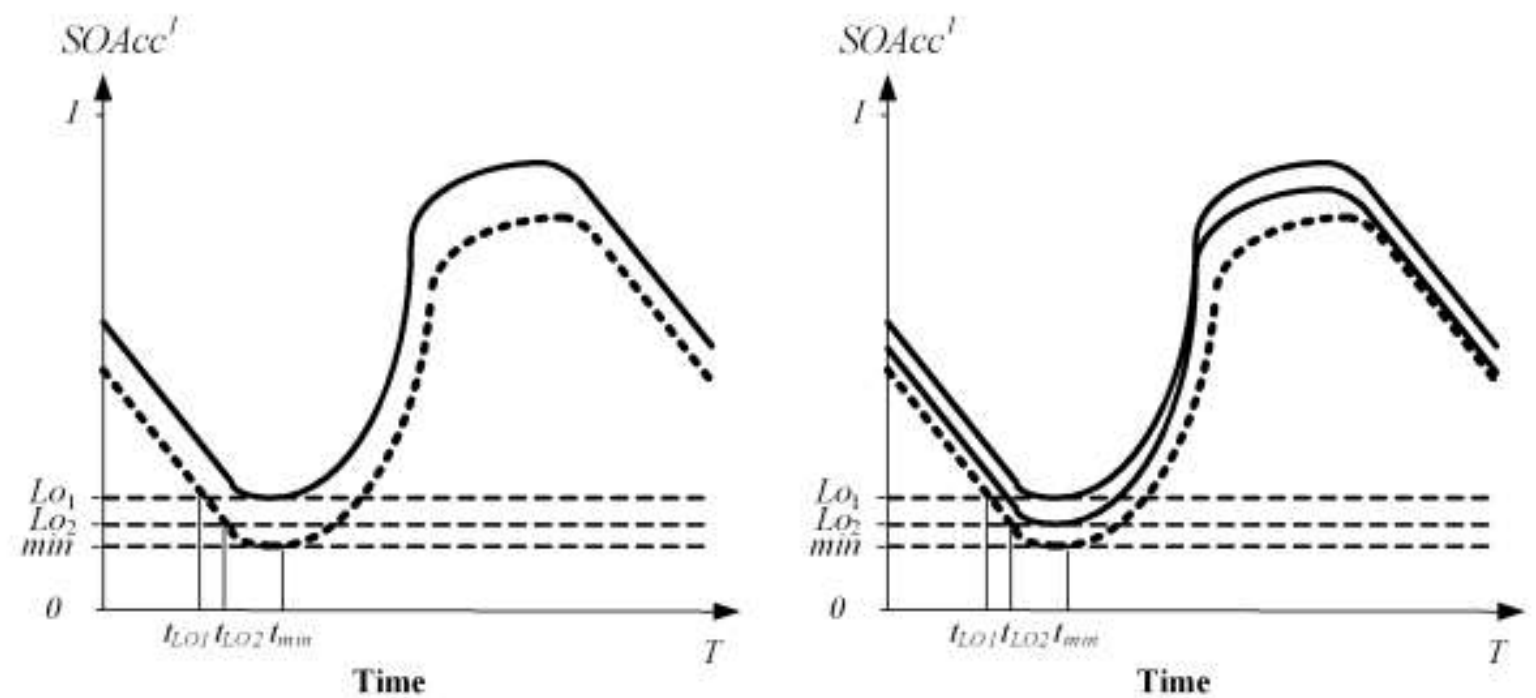

Figure 6: Pinch analysis when multiple Lo limits are imposed.

A third choice (not shown in Fig. 6) can be a shift between $\operatorname{MOES}_{1}^{l, q_{k}}(k)$ and $\operatorname{MOES}_{2}^{l, q_{k}}(k)$; in this case the values of $a_{1}$ and $a_{2}$ are between 0 and 1, it holds that $a_{1}+a_{2}=1$, and the shift is based on the limit $L o=a_{1} \cdot L o_{1}+a_{2} \cdot L o_{2}$. Generalising this scenario it is possible to have $N_{l m}$ limits and therefore:

$$
\begin{aligned}
& \operatorname{MOES}_{c}^{l, q_{k}}(k)=\operatorname{SOAcc}^{l, q_{k}}\left(t_{L O c}\right)-\operatorname{SOAcc}^{l, q_{k}}\left(t_{\text {min }}\right), c \in\left[1, N_{l m}\right] \\
& \operatorname{MOES}^{l, q_{k}}(k)=\sum_{c=1}^{N_{l m}} a_{c} \cdot \operatorname{MOES}_{c}^{l, q_{k}}(k), a_{c} \in[0,1] \\
& \operatorname{SOAcc}_{\text {req }}^{l, q_{k}}\left(t_{0}\right)=\operatorname{SOAcc}_{\text {min }}^{l, q_{k}}\left(t_{0}\right)+\operatorname{MOES}^{l, q_{k}}(k)
\end{aligned}
$$

\section{Case 2: A combination of $L o$ and $U p$ limits}

This is very relevant for the system investigated in this work, where the $U p$ limit may correspond to generally undesired but necessary deactivation of the $P V$ s to protect the $B A T$ from overcharging at the expense of wasting renewable energy in conditions of high solar radiation. In this case, it might also be a priority for the system operator to avoid such a situation which may be addressed considering the following three subcases: 
- In subcase A (Figs. 7a, 7c) the original PGCC starting from a specific $\operatorname{SOAcc}^{l, q_{k}}\left(t_{0}\right)$ violates the two limits ( $L o$ and $U p$ ). The shifted PGCC will either further violate the $U p$ (Fig. $7 \mathrm{~b}$ ) or the Lo limit (Fig. 7d) hence the $\operatorname{SOAcc}_{r e q}^{l, q_{k}}\left(t_{0}\right)$ will be calculated by the following set of equations:

$$
\begin{aligned}
& \operatorname{MOES}^{l, q_{k}}(k)=\operatorname{SOACc}^{l, q_{k}}\left(t_{L O}\right)-\operatorname{SOACc}^{l, q_{k}}\left(t_{\text {min }}\right) \\
& \operatorname{AEEND}^{l, q_{k}}(k)=\operatorname{SOAcc}^{l, q_{k}}\left(t_{\text {max }}\right)-\operatorname{SOAcc}^{l, q_{k}}\left(t_{U p}\right) \\
& \operatorname{SOAcc}_{\text {req }}^{l, q_{k}}\left(t_{0}\right)=\operatorname{SOACc}^{l, q_{k}}\left(t_{0}\right)+a_{1} \cdot \operatorname{MOES}^{l, q_{k}}(k)-a_{2} \cdot \operatorname{AEEND~}^{l, q_{k}}(k)
\end{aligned}
$$

If $a_{1}=1$ and $a_{2}=0$, the $L o$ limit will not be violated but the $U p$ limit will be violated. The same holds for the $L o$ limit if $a_{1}=0$ and $a_{2}=1$. Again it is possible to choose the values of $a_{1}$ and $a_{2}$ between 0 and 1 if an intermediate solution is desired.

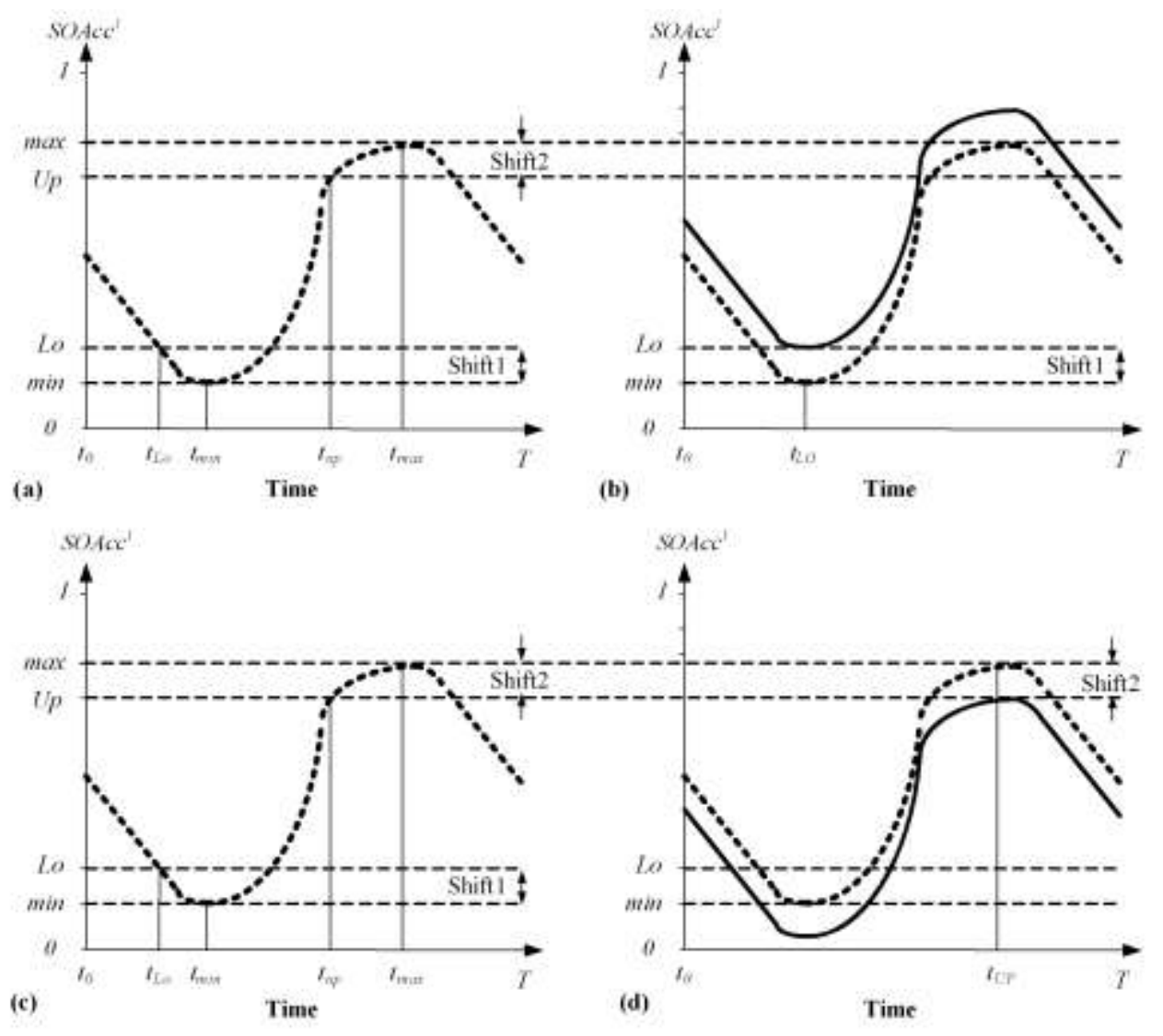

Figure 7: Pinch analysis when a) $L o$ and c) $U p$ limits are imposed; b) The shifted PGCC violates only the $U p$ limit, d) The shifted PGCC violates the $L o$ limit. 
- In subcase B the original PGCC starting from a specific $S O A c c^{l, q_{k}}\left(t_{0}\right)$ violates the Lo limit and not the $U p$ limit but the shifted PGCC violates the $U p$ limit. The equations here are the same as in subcase A.

- In subcase $\mathrm{C}$ the original PGCC starting from a specific $S O A c c^{l, q_{k}}\left(t_{0}\right)$ violates the Lo limit and the shifted PGCC does not violate the $U p$ limit. This case does not require any specific treatment and effectively it is similar to Case 1.

\section{Case 3: Consideration of different energy carriers}

Cases of two energy carriers (e.g., power and hydrogen) are very relevant to hybrid power generation systems such as the one considered in this work. Two different energy carriers are considered in two PGCCs for accumulators $l_{1}$ and $l_{2}$ (Fig. 8). In this case two SOAcc targets must be considered simultaneously in order to satisfy the same operating goal; it is possible to study $S O A c c^{l_{1}}$ first and then $S O A c c^{l_{2}}$ hence avoiding all combinations between the two. As shown in Fig. 8 the PGCCs for the two carriers are decomposed into two diagrams. The two PGCCs start from $\operatorname{SOACc}^{l_{1}, q_{k}}\left(t_{0}\right)$ and $\operatorname{SOACc}^{l_{2}, q_{k}}\left(t_{0}\right)$ and the corresponding $L o$ limits are $L o_{1}$ and $L o_{2}$. In this case the expressions in (8) and (9) are repeated for each SOAcc.

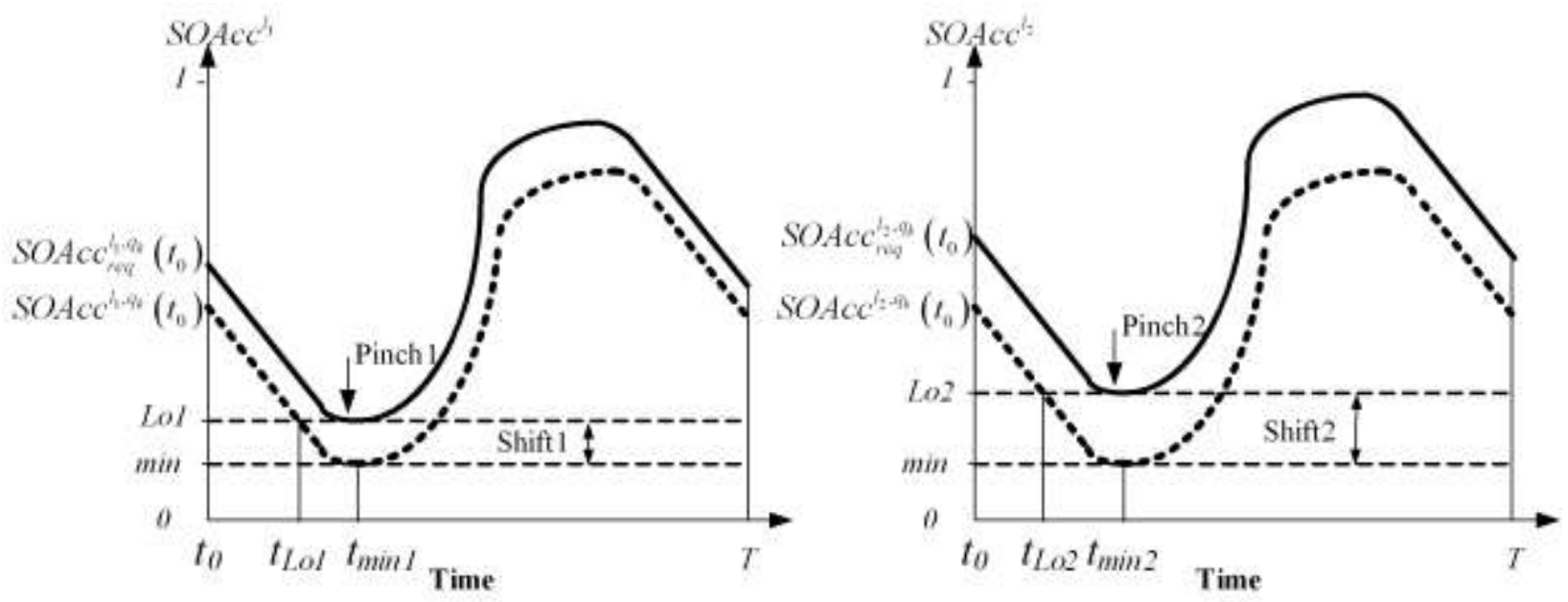

Figure 8: Pinch analysis when multiple energy carriers are considered. 
The above analysis encapsulates all the potential limits that need to be considered during the selection of an appropriate PMS. All these limits reflect on the energy or material that should already be available in order to find a PMS that satisfies the imposed operating goals. Hence, this shows that the proposed approach captures different potential operating policies and priorities. Note that the term "materials" refers to energy carriers which may be treated in a similar way as power and in systems like the one considered in this work. Pinch-based methods have been developed to address water [45] and hydrogen [46] utilization networks considering issues such as purity, but the implementation of these methods are beyond the scope of this work.

\subsection{Selection of PMS for efficient short-term adaptive operation}

In this section the aforementioned analysis is used in order to select the PMS from the set $Q$ that efficiently satisfies the operating goals, based on investigation of subsequent time intervals. Prior to the adaptive implementation of pinch analysis the proposed approach involves the following steps:

- Definition of a set of goals for the hybrid renewable energy system (HRES) (e.g., minimization of the OES).

- Determination of the key HRES variables that impose the largest impact on the system goals (e.g., SOAcc for accumulators).

- Selection of a suitable time prediction horizon $N_{p}$, for which the behaviour of the system can be accurately predicted and the PGCC estimated. The prediction horizon is an integer multiple of the time interval $\Delta T$ used for decision making purposes. The length of the prediction time horizon should be long enough to ensure the satisfaction of the system goals for a selected PMS despite the influence of variations. However, a very long prediction horizon makes the system susceptible to future disturbances.

- Selection of a suitable control time horizon $N_{c}$, for which decisions about the sequence of equipment utilization and operating pattern in the aggregated form of a PMS are taken. The 
control horizon is an integer multiple of the time interval $\Delta T$ used for decision making purposes.

- Identification of a set of potentially efficient PMS for the system.

The implementation of pinch analysis for the efficient adaptive system operation then consists of the following two steps:

- Stored energy targeting: This step involves the identification of the minimum (or maximum for $U p$ limits) amount of stored energy (in various forms) that should be recovered from internal resources at the end of the control horizon $\left(k+N_{c^{-}} 1\right) \Delta T$ to serve the operating goals during the prediction horizon $\left(k+N_{p}-1\right) \Delta T$ (i.e. avoid using external resources, avoid undesired de-activation of particular converters etc.).

- Target matching: This step involves the identification of the employed PMS during the control horizon $\left(k+N_{c}-1\right) \Delta T$ in order to meet the target for stored energy identified during the prediction time horizon $\left(k+N_{p^{-}}-1\right) \Delta T$ starting from $S O A c c^{l, q k}\left(t_{0}\right)$.

To clearly elaborate the proposed approach it is assumed for the prediction horizon that $N_{p}=2$ and for the control horizon that $N_{c}=1$. In this respect, for the prediction time horizon $k+1$ the PGCC is computed for each PMS for all possible initial SOACc values, based on weather forecasts and historical load data. Fig. 9 shows an example with three PMS (i.e. $p_{k+1}, q_{k+1}, h_{k+1} \in Q$ ) for the initial SOACc values that produced the lowest $S O A c c\left(t_{\min }\right)$ for each PMS. It is observed that different PMS result in different PGCCs and different outsourced electricity supply (MOES) requirements for the system, i.e. in this case $\operatorname{MOES}^{l, p_{k+1}}(k+1)=0$, and $\operatorname{MOES}^{l, q_{k+1}}(k+1)>\operatorname{MOES}^{l, h_{k+1}}(k+1)$. Since the goal in the stored energy targeting step is to develop a sufficient inventory in $k$ so that the system may operate autonomously in $k+1$ (i.e. without the use of non-renewable resources) the following target is imposed:

$$
\operatorname{SOACc}_{T A R}^{l}(k+1)=\max _{q \in Q} \operatorname{SOACc}_{r e q}^{l, q} k+1\left(t_{0}\right), q \in Q
$$




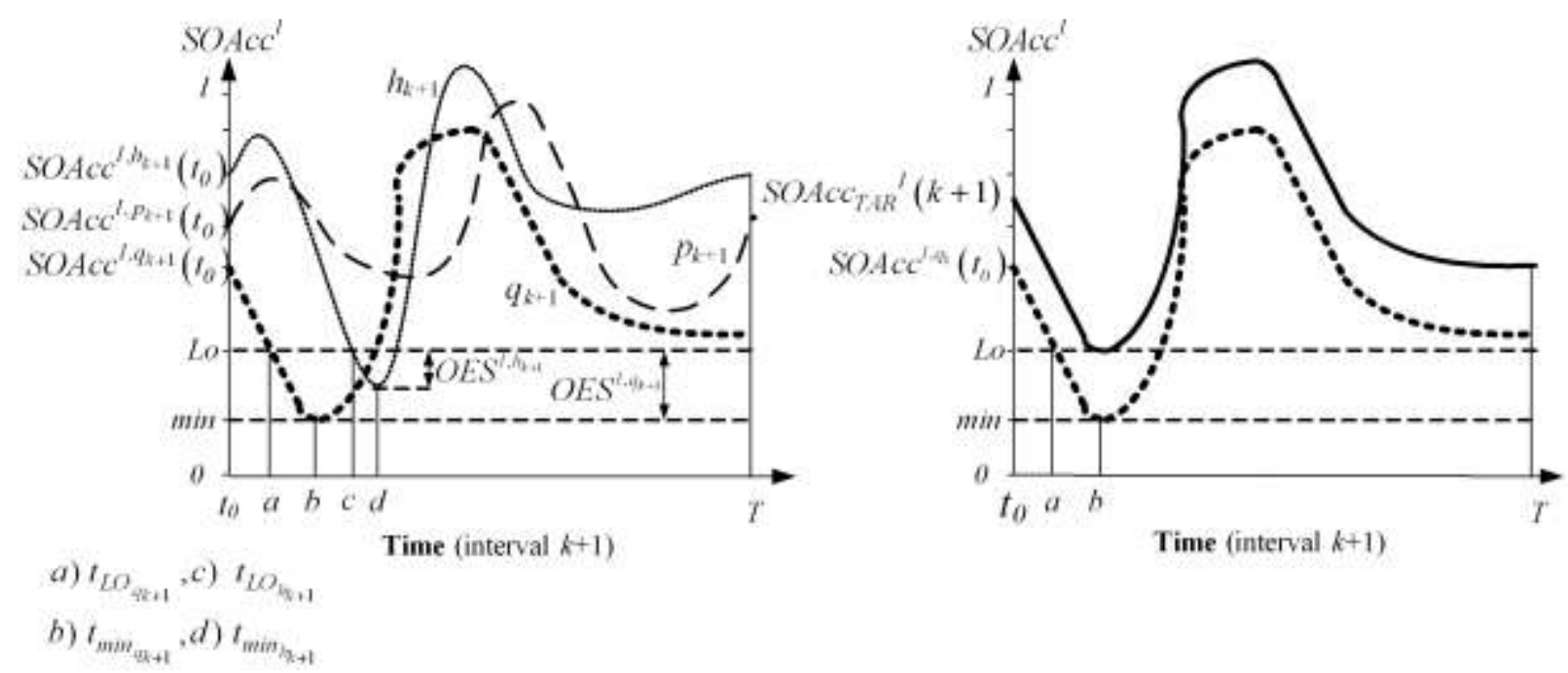

Figure 9: Location of the target value identified in interval $k+1$; a) shows the PGCC of 3 different PMS and the corresponding extra energy that is required, b) shows the shifted PGCC of the PMS that requires the largest shift.

Equation (14) indicates the amount of energy that needs to become available at $t=T$ in control horizon $k$ in order to guarantee that regardless of the PMS that is chosen during the future prediction horizon $S O A c c^{l, q} k+1$ it will never violate the limit $L o$, meaning that the required zero MOES is satisfied. Note that this analysis addresses the development of an appropriate inventory in equipment of pre-specified capacity. The capacity is assumed to guarantee system controllability, in the sense that the considered variability can be compensated by the PMS. The same analysis may potentially be used for the sizing of equipment, but this was not further investigated. Obviously, SOAcc $c_{r e q}^{l, q+1}\left(t_{0}\right)$ may be calculated based on the analysis presented in sections 3.1 and 3.3, depending on the system type that is examined. The "max" operator indicates that the PMS resulting in the largest shift should be selected corresponding to the worst-case scenario. This serves the goal of avoiding the use of external energy input, which is of major importance in hybrid systems. However, the trade-offs investigated in section 3 among different operating goals and limits may also be considered in the determination of $\operatorname{SOAcc}_{T A R}^{l}(k+1)$. 
Potential cases that may be investigated combining the analysis of section 3 with equation (14) involve the following:

- If there are two (or multiple) values of $L o$ limits then a decision has to be taken regarding which one to choose considering the analysis in Case 1 of section 3.3.

- If by shifting the PGCC there is an upper limit violation a decision must be taken which one to satisfy considering the analysis in Case 2 of section 3.3.

- If there are multiple $S O A c c^{l}$ values that need to be satisfied, then an expression such as equation (14) must be produced for each $S O A c c^{l}$ considering the analysis in Case 3 of section 3.3 .

Closer observation of Fig. 9 indicates that it is possible to consider fewer PMS in equation (14) without having to calculate all of them, prior to identifying the one that will result in the desired shift at interval $k+1$. In the case considered in Fig. 9 to illustrate the proposed approach it is observed that there is one valley (at $t=t_{\min }$ ) and one peak value (at $t=t_{u p}$ ) for $S O A c c^{l}$ in each PMS. Apparently, in the interval $\left[0, t_{\min }\right]$ there should be no usage of converters that reduce the value of SOAcc ${ }^{l}$ (i.e. $\varepsilon_{t, l \rightarrow m}=0$ ) and in the interval $\left[t_{\min }, t_{\max }\right]$ there should be no activation of converters that supply the accumulator $l$ (i.e. $\varepsilon_{t, m \rightarrow l}=0$ ) apart from the RES ones. Hence, PMS that violate this condition can be excluded from the set $Q$ (the same analysis may be extended to more complex cases). Such PMS will be part of a set $Q_{S} \subseteq Q$ which may be identified as follows:

$$
\begin{aligned}
& \operatorname{SOACc}_{T A R}^{l}(k+1)=\max _{q \in Q_{S}} \operatorname{SOAcc}_{r e q}^{l, q_{k+1}}\left(t_{0}\right), q \in Q_{s} \\
& Q_{s}=\left\{\begin{array}{l}
q_{k+1}:\left[\varepsilon_{t, l \rightarrow m}=0 \forall t \in\left[0, t_{\min }\right]\right] \wedge\left[\varepsilon_{t, m \rightarrow l}=0 \forall t \in\left[t_{\min }, t_{\max }\right]\right], \\
\forall q \in Q, l \in R s^{A c c}, m \in R s
\end{array}\right\}
\end{aligned}
$$

In the stored energy targeting step a specific target has been set for $\operatorname{SOAcc}^{l, q_{k+1}}\left(t_{0}\right), l \in R^{A c c}, q_{k+1} \in Q$ for each accumulator. This target is effectively the final 
value of $\operatorname{SOAcc}^{l}$ at the end of the control horizon $k$, i.e. $\operatorname{SOAcc}^{l, q_{k+1}}\left(t_{0}\right)=\operatorname{SOAcc}^{l, q} k(T)$. In the target matching step, for control horizon $k$, a PMS is sought that results in a $\operatorname{SOAcc}^{l, q} k(T)$ as close as possible to $\operatorname{SOAcc}_{T A R}^{l}(k+1)$. This is expressed as follows:

$$
q_{k}^{o p t}=\left(\min _{q_{k} \in Q}\left(\left|\operatorname{SOAcc}_{T A R}^{l}(k+1)-\operatorname{SOAcc}^{l, q} k(T)\right|\right)\right) \wedge\left(\operatorname{SOAcc}^{l, q} k(T)>\operatorname{SOAcc}_{T A R}^{l}(k+1)\right)
$$

The entire procedure is illustrated in Fig. 10 where the PGCC has been shifted first in the prediction horizon $k+1$ and then from implementation of equations (14)-(17) it was found that $q_{k}^{o p t}=q_{k}$. It is important to note here that the operating zones of the converters at the beginning of the control horizon $k$ must be set according to the desired location that was found for the previous implementation of the algorithm. After the procedure ends, the time horizons shift forward in time by one decision making interval and the procedure is repeated.

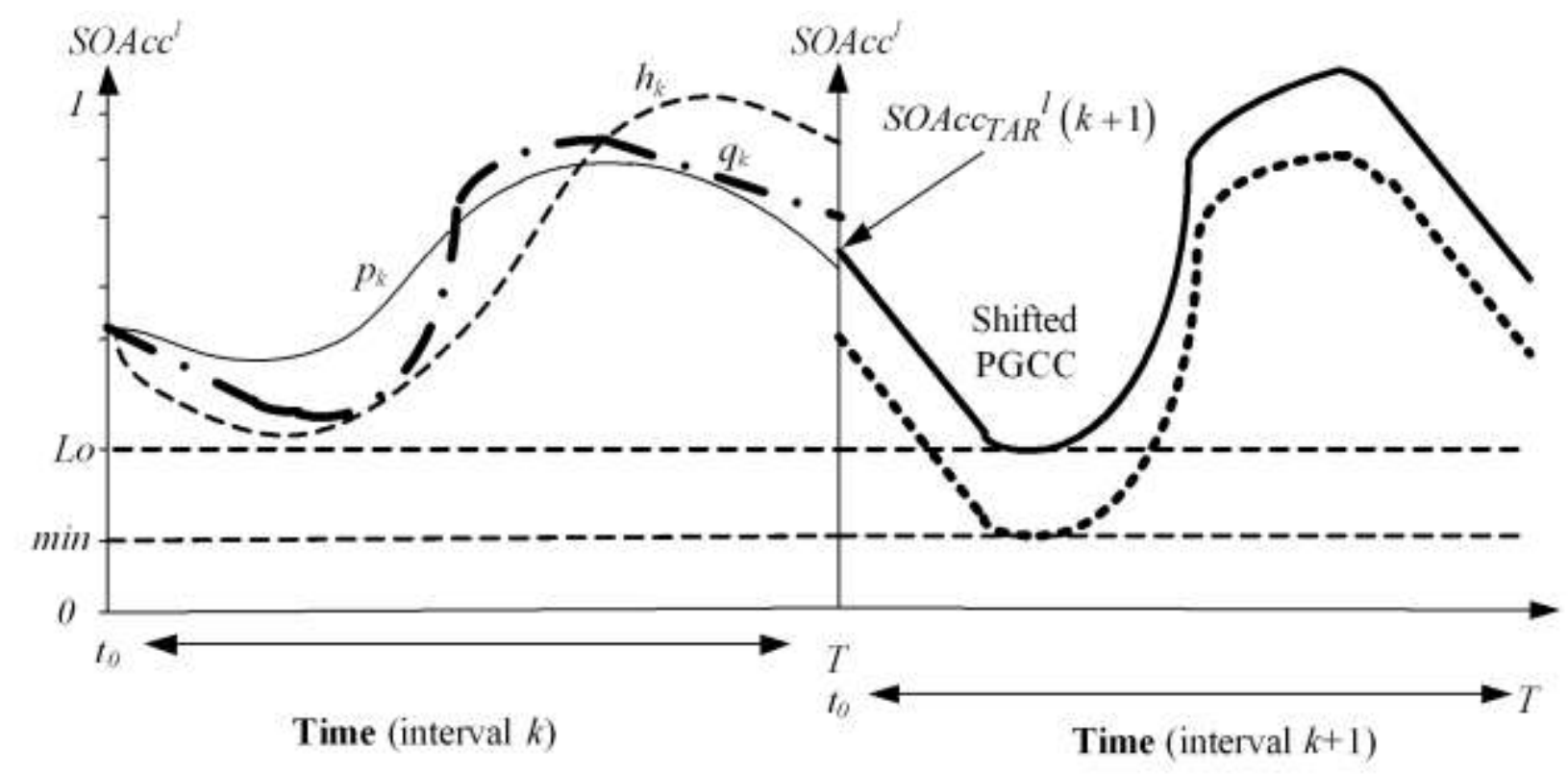

Figure 10: Selection of most efficient PMS; the selected PGCC and its corresponding shift are shown in interval $k+1$ and the PGCC of three available PMS and the corresponding final value are shown in the interval $k$. 
Care has to be taken if there are multiple targets that need to be satisfied in control horizon $k$ (as in case 3 of the previous section), i.e. there exist multiple values such as $\operatorname{SOACc}_{T A R}^{l_{1}}(k+1), \operatorname{SOAcc}_{T A R}^{l_{2}}(k+1), \operatorname{SOACc}_{T A R}^{l_{3}}(k+1)$ and so forth. In this case the above procedure must be repeated for each $S O A c c^{l}$ which will result in the following cases:

a. Only one PMS that satisfies all targets and this is chosen for horizon $k$.

b. Multiple PMSs that satisfy all targets; for example, assume a case with three targets $\operatorname{SOAcc}_{T A R}^{l}(k+1), l \in\left\{l_{1}, l_{2}, l_{3}\right\}$ and two PMSs $\left(h_{k}\right.$ and $\left.p_{k}\right)$ that satisfy these targets. Then the chosen PMS is the one that satisfies the following condition:

$$
\min _{q_{k} \in\left\{h_{k}, p_{k}\right\}}\left(\operatorname{SOAcc}^{l, q_{k}}(T)-\operatorname{SOAcc}_{T A R}^{l}(k+1)\right), l \in\left[l_{1}, l_{2}\right]
$$

c. No PMS satisfies all conditions simultaneously; then it is possible to use a condition as above or to choose the PMS that satisfies a desired target.

Note that the symbol $q_{k}^{o p t}$ of equation (17) implies the selected PMS which best matches the stored energy target and hence satisfies the desired operating goal. However, it should be clarified that the aim of this work is not to identify the parameter values, decision sequences or operating constraints that determine an optimum PMS. This has been studied in numerous publications and there is a wide variety of PMS available. Instead in this work it is assumed that there pre-exists a set of PMS (e.g., from the relevant literature) that enable efficient utilization of the available resources and equipment to meet specific targets within specific operating constraints. We therefore propose a method to identify which of these PMS to use in a given interval. The criterion that determines the selected PMS is the satisfaction of a single or multiple desired operating goals. The method involves a systematic and constant alteration of the PMS that is used in order to have the best possible performance under the inherent variability of such systems. It is not implemented using an optimisation algorithm but instead the user defines the allowable levels of energy storage 
and then a graphical representation is employed based on pinch analysis, in order to choose the most suitable PMS that enables operation within specific constraints.

\subsection{Algorithmic implementation}

The analysis presented in the previous sections may be implemented in the form of an algorithmic sequence which integrates the proposed equations. Let $\left[L \sigma_{m \rightarrow l}^{\text {SOAcc }}(t), U p_{m \rightarrow l}^{\text {SOAcc }}(t)\right]$ $\forall q \in\left\{P M S_{1}, \ldots, P M S_{N}\right\}, \forall t \in\left[t_{0}, T\right]$ be a range of operating limits for the activation or deactivation of a converter $m$ based on the state of an accumulator $l$ for a maximum of $N$ available

PMS, and let $\left[v^{L}, v^{H}\right]$ be a range of initial values for the SOAcc of accumulator $l$. The two steps involving stored energy targeting and target matching of section 3.4 are implemented in the following 2 iterations:

1. For the prediction horizon $k+1$ :

1.2 For each $q_{k+1} \in\left\{P M S_{1}, \ldots, P M S_{N}\right\}$ :

1.2.1 Select a set of $S O A c c^{l, q_{k+1}}\left(t_{0}\right)$ values in the range $\left[v^{L}, v^{H}\right]$ with a desired step $d v$.

1.2.2 For each $\quad \operatorname{SOACC}^{l, q_{k+1}}\left(t_{0}\right) \in\left[v^{L}, v^{H}\right] \quad$ using (1)-(5) calculate $\operatorname{SOACc}^{l, q_{k+1}}(t) \forall t \in\left[t_{0}, T\right]$ with a desired step $d t$.

1.2.2.1 From equation (10a) calculate $\operatorname{SOACc}^{l, q_{k+1}}\left(t_{\min }\right)$.

1.2.2.2 From equation (6) calculate $O E S^{l, q_{k+1}}(k+1)$

1.2.3 From equation (8b) or (12a) or (13a) calculate $\operatorname{MOES}^{l, q_{k+1}}(k+1)$ depending on the case that is investigated. 
1.2.4 From equation (10b) calculate $S O A C c_{\min }^{l, q_{k+1}}\left(t_{0}\right)$. In case there are multiple $\operatorname{SOACc}_{\min }^{l, q_{k+1}}\left(t_{0}\right)$ as a result of steps 1.2.2 and 1.2.3 (i.e. equation (10b) is satisfied for more than one $\left.O E S^{l, q_{k+1}}(k+1)\right)$, use the maximum one.

1.2.5 From equation (9) or (12b) or $(13 \mathrm{~b}, \mathrm{c})$ calculate $S O A c c_{r e q}^{l, q_{k+1}}\left(t_{0}\right)$ depending on the case that is investigated.

1.3 From equation (14) or (15) subject to (16) calculate $\operatorname{SOAcc}_{T A R}^{l}(k+1)$.

2. For the control horizon $k$, let an initial pre-specified state of the accumulator $l, S O A c c^{l, q_{k}}\left(t_{0}\right)$ $\forall q_{k} \in\left\{P M S_{1}, \ldots, P M S_{N}\right\}$ and let the same operating limits as in the prediction horizon $k+1$ :

2.1 Set $\left[\operatorname{Lo}_{m \rightarrow l}^{\operatorname{SOAcc}^{l}}(t)+\operatorname{MOES}^{l, q_{k}}(k), U p_{m \rightarrow l}^{\text {SOAccl}^{l}}(t)+\operatorname{MOES}^{l, q_{k}}(k)\right] \forall q \in\left\{P M S_{1}, \ldots, P M S_{N}\right\}$.

2.2 For $q_{k} \in\left\{P M S_{1}, \ldots, P M S_{N}\right\}$ :

2.1.1 From equations (1)-(5) calculate $S O A c c^{l, q_{k}}(T)$

2.3 From equation (17) identify $q_{k}^{\text {opt }}$.

Note that $\operatorname{MOES}^{l, q_{1}}(1)$ is initialized to zero, i.e. when iterations 1 and 2 are implemented for $k=1$. When they are implemented for $k=2$ then $\operatorname{MOES}^{l, q_{2}}(2)$ is already available from the previous implementation of iteration 1 for $k=1$ and so forth for $\mathrm{k}>2$. The need for the shift in the $L o$ and $U p$ limits has been elaborated in section 3.2 and should not be implemented when it is desired to avoid the activation of a particular device. Furthermore, the initial state of the accumulator $l$ at $k=2$ and $t_{0}$ becomes $\operatorname{SOAcc}^{l, q_{2}}\left(t_{0}\right)=\operatorname{SOACc}^{l, q_{1}}(T)$ and so forth for $\mathrm{k}>2$. 


\section{Implementation}

\subsection{Power management strategies}

This section presents issues associated with the implementation of the proposed adaptive management method in the isolated microgrid described in section 3. The set $Q$ consists of 9 PMSs, 4 of them are thoroughly described in [1] and the other 5 are derived for the first time in this work based on the previous 4. More specifically, the 9 PMS are based on PMS 1 presented in [1] which is described briefly below and may be mathematically represented using equations (1)-

1. The $F C$ will be operated if:
a. $S O A c c^{B A T}<L o_{F C \rightarrow B A T}^{S O A c B A T}(t)$ and there is a hysteresis zone until $U p_{F C \rightarrow B A T}^{S O A c c^{B A T}}(t)$.
b. $S O A c c^{F T}>L o_{F C \rightarrow B A T}^{S O A c C^{F T}}(t)$ without a hysteresis zone.
c. $S O A c c^{W T}>\operatorname{LO}_{F C \rightarrow B A T}^{S O A c C^{W T}}(t)$ without a hysteresis zone.

2. The $E L$ will be operated if:
a. $S O A C^{B A T}>U p_{B A T \rightarrow E L}^{S O A c^{B A T}}(t)$ and there is a hysteresis zone until $\operatorname{Lo}_{B A T \rightarrow E L}^{S O A c^{B A T}}(t)$.
b. $S O A c c^{W T}>\operatorname{Lo}_{B A T \rightarrow E L}^{S O A c c^{W T}}(t)$ without a hysteresis zone.
c. $S O A c c^{B F}<\operatorname{Lo}_{B A T \rightarrow E L}^{S O A c^{B F}}(t)$ without a hysteresis zone.

3. The DSL will be operated if $S O A c c^{B A T}>\operatorname{Lo} o_{D S L \rightarrow B A C c^{B A T}}^{S O A}(t)$ and there is a hysteresis zone until $U p_{D S L \rightarrow B A T}^{S O A c c^{B A T}}(t)$

4. The $P V$ will be operated if $S O A c c^{B A T}<\operatorname{Lo}_{P V \rightarrow B A T}^{S O A c^{B A T}}(t)$ without a hysteresis zone.

5. The $W G$ will be operated if $S O A c c^{B A T}<\operatorname{Lo}_{W G \rightarrow B A T}^{S O A c c^{B A T}}(t)$ without a hysteresis zone.

In order to produce the other 8 PMSs (shown in Table 1) the following combinations for the hysteresis zone and power produced/consumed by the $F C$ and $E L$ take place: 
A. The hysteresis zones (see also Fig. 11):

i. Are fixed, i.e. $L o_{F C \rightarrow B A T}^{S O A c c^{B A T}}(t), \operatorname{Lo}_{B A T \rightarrow E L}^{S O A c c^{B A T}}(t), U p_{F C \rightarrow B A T}^{S O A c^{B A T}}(t)$, and $U p_{B A T \rightarrow E L}^{S O A c c^{B A T}}(t)$ are fixed throughout the operation of the system.

ii. Depend on the time of the year, i.e. during the months with high solar irradiation $U p_{F C \rightarrow B A T}^{S O A c c^{B A T}}(t)=\operatorname{Lo}_{F C \rightarrow B A T}^{S O A c c^{B A T}}(t)$ and during the months with low solar irradiation $\operatorname{Lo}_{B A T \rightarrow E L}^{S O A c c^{B A T}}(t)=U p_{B A T \rightarrow E L}^{S O A c c^{B A T}}(t)$

iii. Depend on the time interval where the devices are activated, i.e. if a device is active for more than a predefined amount of time then $U p_{F C \rightarrow B A T}^{S O A c c^{B A T}}(t)=\operatorname{Lo}_{F C \rightarrow B A T}^{S O A c c^{B A T}}(t)$ and $\operatorname{Lo}_{B A T \rightarrow E L}^{S O A C c^{B A T}}(t)=U p_{B A T \rightarrow E L}^{S O A c c^{B A T}}(t)$

B. The power produced by the RES is greater (i.e. surplus) or less (i.e. deficit) than the power requested by the $L D$, i.e. $P(t)=F_{P V \rightarrow B A T}^{\text {Out,Pow }}(t)+F_{W G \rightarrow B A T}^{\text {Out, Pow }}(t)-F_{B A T \rightarrow L D}^{\text {Out,Pow }}(t)$ :

i. $\quad P(t)$ is not known and the power of the $F C$ and $E L$ are fixed (at rated values).

ii. $P(t)$ is not known and the power of the $F C$ and $E L$ are a linear function of the SOAcc ${ }^{B A T}$, i.e. $\quad F_{F C \rightarrow B A T}^{\text {Out,Pow }}(t)=a_{F C} \cdot S O A c c^{B A T}(t)+b_{F C} \quad$ and $F_{B A T \rightarrow E L}^{\text {Out } P \text { Pow }}(t)=a_{E L} \cdot \operatorname{SOAcc}^{B A T}(t)+b_{E L}$ where $a_{F C}, a_{E L}, b_{F C}$ and $b_{E L}$ are coefficients that depend on the operational range of the devices.

iii. $P(t)$ is known and the power produced by the $F C$ (and the power used by the $E L$ ) equals this power deficit (surplus), i.e.

$$
\begin{aligned}
& F_{F C \rightarrow B A T}^{\text {Out }, P \text { Pow }}(t)=F_{B A T \rightarrow L D}^{I n, P O w}(t)-F_{P V \rightarrow B A T}^{\text {Out } P \text { ow }}(t)-F_{W G \rightarrow B A T}^{\text {Out,Pow }}(t) \text { and } \\
& F_{B A T \rightarrow E L}^{\text {Out,Pow }}(t)=F_{P V \rightarrow B A T}^{\text {Out,Pow }}(t)+F_{W G \rightarrow B A T}^{\text {Out,Pow }}(t)-F_{B A T \rightarrow L D}^{I n, P o w}(t)
\end{aligned}
$$




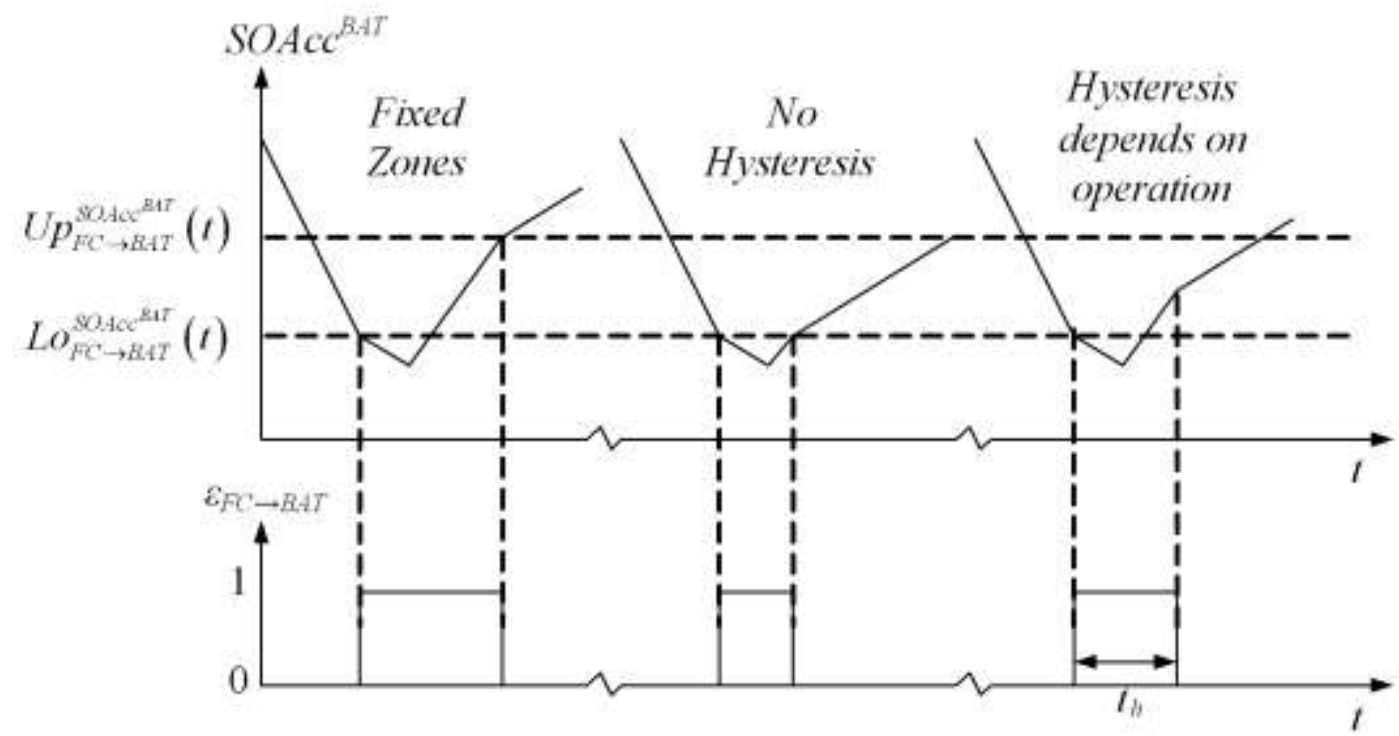

Figure 11: Examples of hysteresis zones.

Table 1: Summary of PMS studied in this work [1]

\begin{tabular}{ccccc}
\hline \multicolumn{5}{c}{ Hysteresis Zone } \\
\hline & Case & $\mathrm{A}(\mathrm{i})$ & $\mathrm{A}(\mathrm{ii})$ & $\mathrm{A}(\mathrm{iii})$ \\
\cline { 2 - 5 } & $\mathrm{B}(\mathrm{i})$ & $\mathrm{PMS}_{1}$ & $\mathrm{PMS}_{4}$ & $\mathrm{PMS}_{7}$ \\
\cline { 3 - 4 } & $\mathrm{B}(\mathrm{ii})$ & $\mathrm{PMS}_{2}$ & $\mathrm{PMS}_{5}$ & $\mathrm{PMS}_{8}$ \\
\hline
\end{tabular}

\subsection{Results and discussion}

\subsubsection{Case considering a Lo limit}

In this subsection we present the results of the proposed method for one Lo limit, considering the 9 PMSs previously presented (i.e. $Q=\left\{P M S_{i, k}\right\}, i \in[1,9], k \in[1,365]$ ). The goal is to ensure that the $S O A c c^{B A T}$ will not drop below $L o=0.2$ (i.e. $\operatorname{SOACc}^{B A T, q_{1}}\left(t_{L o}\right) \geq 0.2$ ) and as a case study the method is tested considering day 1 as the control horizon ( $1^{\text {st }}$ of January) and day 2 as the prediction horizon. The $L D$ demand is assumed to be constant at $2 \mathrm{~kW}$. The remaining parameters (e.g., size of $B A T, F T$ and so forth) are described in [1]. To find the PMS that best satisfies the above goal in day 1 we investigate the power generation profile of day 2 considering the 9 PMSs for a range of 
initial values of $S O A c c^{B A T}$. From section 3.5 for $k=2$ the operating regions of the $F C$ and $D S L$ are set at $\left[\operatorname{Lo}_{F C \rightarrow B A T}^{S O A C c^{B A T}}(t), U p_{F C \rightarrow B A T}^{S O A C c^{B A T}}(t)\right]=[0.3,0.35]$ and $\left[\operatorname{Lo}_{D S L \rightarrow B A T}^{S O A c c^{B A T}}(t), U p_{D S L \rightarrow B A T}^{S O A c c^{B A T}}(t)\right]=[0.2,0.205]$ $\forall q_{2} \in\left\{P M S_{1}, \ldots, P M S_{9}\right\}, \forall t \in[1,24]$. For each PMS the PGCC is calculated for a range of initial values of $S O A c c^{B A T, q_{2}}(1) \in[0.2,0.8]$ with a step size of $d v=0.001$. This generates 600 different PGCC profiles for each PMS or 5400 in total and the lowest $S O A c c^{B A T, q_{2}}\left(t_{\text {min }}\right)$ together with $\operatorname{OES}^{B A T, q_{2}}(2)$ are recorded. As a result we find the largest shift (i.e. $\operatorname{MOES}^{B A T, q_{2}}(2)$ ) that is required for each PMS. In order to improve the readability of the paper, initially, in Table 2 and Fig. 12 we see the aforementioned analysis for the first 2 PMSs. At this point it has to be mentioned that due to the complexity of the system it is possible to have multiple values for $S O A c c^{B A T}$ (1) that produce the same $\operatorname{SOAcc}^{B A T}\left(t_{\min }\right)$ (see row 4 of Table 2) and the maximum one in each case is selected (see row 5 of Table 2). Hence using equation (9) for each PMS it is possible to find the largest value of $\operatorname{SOAcc}^{B A T}(2)$ that is required in order to ensure that if a specific PMS is selected in day 2 the $S O A c c^{B A T}$ will drop below 0.2 (row 6). Based on equation (14) and by taking the maximum of these values (row 7) we find the target value for day 2 .

Table 2: $S O A c c^{B A T}$ values considered in the case study

\begin{tabular}{lll}
\hline & PMS1 & PMS2 \\
\hline $\operatorname{SOAcc}^{B A T}\left(t_{\text {min }}\right)$ & 0.184 & 0.193 \\
\hline $\operatorname{MOES}^{B A T, q_{2}}(2)$ & $0.2-0.184=0.016$ & $0.2-0.193=0.007$ \\
\hline $\operatorname{SOAcc}_{\min }^{B A T, q_{2}}(1)$ & $0.201,0.209,0.225$ & $0.208,0.232$ \\
\hline $\max \left(\operatorname{SOAcc}_{\min }^{B A T, q_{i}}(1)\right), i \in Q$ & 0.225 & 0.232 \\
\hline $\operatorname{SOAcc}_{\mathrm{req}}^{B A T, q_{2}}(1)$ & $0.225+0.2-0.184=0.241$ & $0.232+0.2-0.193=0.239$ \\
\hline $\operatorname{SOAcc}_{\text {TAR }}^{B A T}(2)$ & 0.241 & \\
\hline
\end{tabular}

Based on the analysis for day 2, the chosen PMS for day 1 is the one that will generate a final value of the $S O A c c^{B A T}$ as close to 0.241 as possible. The latter is the level of charge that should be stored in the battery in day 1 in order to avoid using the DSL in day 2. It is possible to calculate 
the PGCC for day 1 and starting from the given initial conditions we see that $\mathrm{PMS}_{1}$ will produce the best result and a final $S O A c c^{B A T}$ at 0.2790 while the $F T$ will be at 0.561 . The complete results for the 9 PMSs are shown in Table 3.

(a)

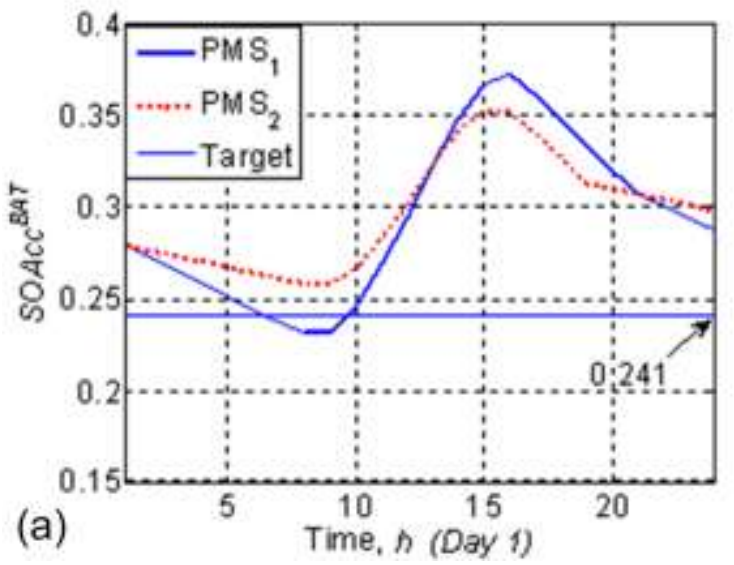

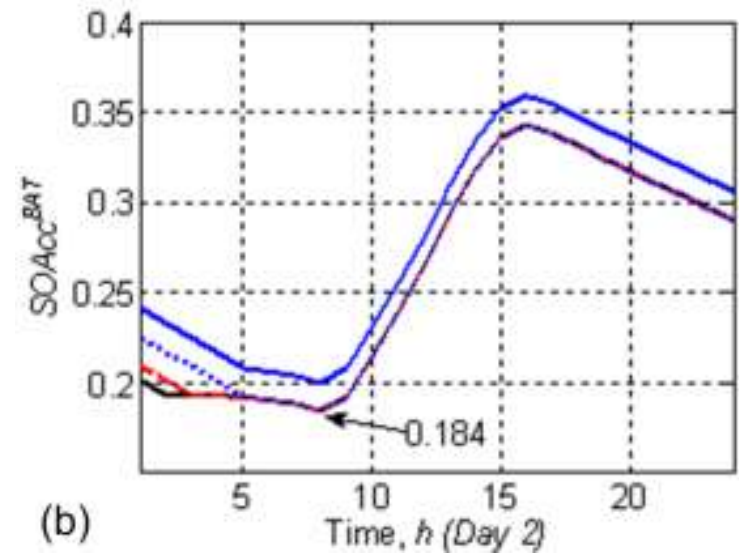

(b)

Figure 12: a) PGCC for day 1 for PMS1 and PMS2, b) Initial and shifted PGCC for PMS1 in day 2.

Table 3: Complete results of case study 1

\begin{tabular}{|c|c|c|c|c|c|c|c|c|c|}
\hline & $\mathrm{PMS}_{1}$ & $\mathrm{PMS}_{2}$ & $\mathrm{PMS}_{3}$ & $\mathrm{PMS}_{4}$ & $\mathrm{PMS}_{5}$ & $\mathrm{PMS}_{6}$ & $\mathrm{PMS}_{7}$ & $\mathrm{PMS}_{8}$ & $\mathrm{PMS}_{9}$ \\
\hline 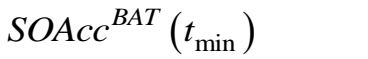 & 0.184 & 0.193 & 0.193 & 0.184 & 0.193 & 0.193 & 0.184 & 0.193 & 0.193 \\
\hline $\operatorname{MOES}^{B A T, q_{2}}(2)$ & 0.016 & 0.007 & 0.007 & 0.016 & 0.007 & 0.007 & 0.016 & 0.007 & 0.007 \\
\hline$S O A c c_{\min }^{B A T, q_{2}}(1)$ & $\begin{array}{l}0.201 \\
0.209, \\
0.225\end{array}$ & $\begin{array}{l}0.208, \\
0.232\end{array}$ & $\begin{array}{l}0.208 \\
0.232\end{array}$ & $\begin{array}{l}0.201 \\
0.209 \\
0.225\end{array}$ & $\begin{array}{l}0.208 \\
0.232\end{array}$ & $\begin{array}{l}0.208 \\
0.232\end{array}$ & $\begin{array}{l}0.201 \\
0.209, \\
0.225\end{array}$ & $\begin{array}{l}0.208 \\
0.232\end{array}$ & $\begin{array}{l}0.208, \\
0.232\end{array}$ \\
\hline $\max \left(S O A c c_{\min }^{B A T, q_{i}}(1)\right)$ & 0.225 & 0.232 & 0.232 & 0.225 & 0.232 & 0.232 & 0.225 & 0.232 & 0.232 \\
\hline$S O A c c_{\text {req }}^{B A T, q_{2}}(1)$ & 0.241 & 0.239 & 0.239 & 0.241 & 0.239 & 0.239 & 0.241 & 0.239 & 0.239 \\
\hline $\operatorname{SOAcc}_{T A R}^{B A T}(2)$ & 0.241 & & & & & & & & \\
\hline
\end{tabular}

Fig. 13 depicts the responses of the 9 PMSs for day 1 and the values of the $S O A c c^{B A T}$ at $t=24$. From these it can be observed that PMS 4 results closest to the target value of 0.241 . An interesting question can be raised here, "why not use PMS 3, as at $t=24$, the SOAcc ${ }^{B A T}$ will have a higher value than when PMS 4 is used, i.e. more available power for the next day?. In order to answer this question it is necessary to remember that the target value for day 2 was set based on the assumption that if it is reached (at the end of day 1) then under normal weather conditions, the $S O A c c^{B A T}$ will not drop below the limit $L o$ (which of course can be set to any value, in case we want to avoid problems associated with deep discharging). Hence, when we get to much higher 
values than the required one, we extend the usage of the $F C$ and hence this decreases the overall efficiency (apart of course from removing hydrogen from the FT that could have been used later). In the above case study using PMS 3 the $F C$ supplied the system with $19.5 \mathrm{kWh}$ while in PMS 1 it supplied $7 \mathrm{kWh}$. This implies that the $F C$ was overused by $15 \%$ when the system was controlled by PMS 3.

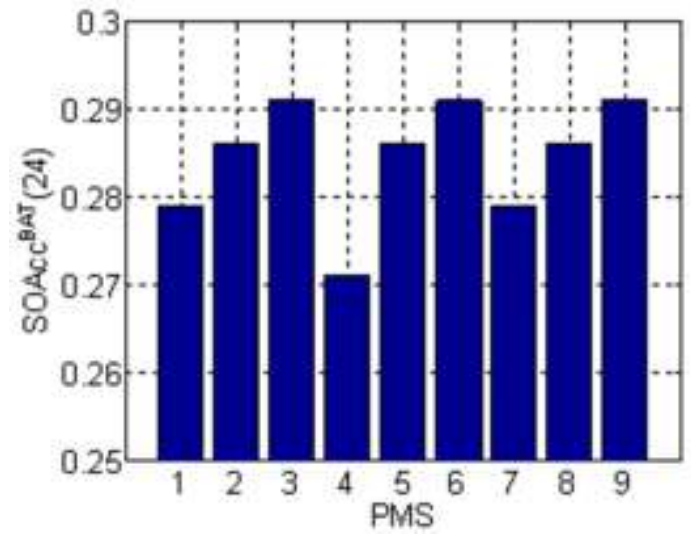

Figure 13: Last value of $S O A c c^{B A T}$ in day 1 for the 9 PMSs for case study 1.

Note that the above steps describe how to identify the most efficient PMS for $k=1$ at iterations 1 and 2. If it was desired to extend the analysis and to identify the most efficient for $k=2$, the two iterations would be repeated by implementing the same procedure for $k=3$ and then for $k=2$. In this case, at $k=2$ the only change compared to the first two iterations is that we need to calculate the operating regions of the $F C$ and $D S L$ at $k=2$ as $\left[\operatorname{Lo}_{F C \rightarrow B A T}^{S O A c c^{B A T}}(t), U p_{F C \rightarrow B A T}^{S O A c c^{B A T}}(t)\right]=$ $[0.3,0.35]+\operatorname{MOES}^{B A T, q_{2}}(2) \quad$ and $\quad\left[\operatorname{Lo}_{D S L \rightarrow B A T}^{S O A c c^{B A T}}(t), U p_{D S L \rightarrow B A T}^{S O A c C^{B A T}}(t)\right]=[0.2,0.205]+M O E S^{B A T, q_{2}}(2)$ with $\operatorname{MOES}^{B A T, q_{2}}(2)$ taken from the previous calculations for $k=1$ and $k=2$. Furthermore, the initial state of the accumulators at $k=2$ and $t_{0}=1$ would now be $\operatorname{SOACc}^{l, q_{2}}\left(t_{0}\right)=\operatorname{SOACc}^{l, q_{1}}(24), \forall l \in\{B A T, F T, B F, W T\}$.

\subsubsection{Case considering two energy carriers}

In this section we present the results when there is a Lo type limit for the BAT and also another Lo type limit for the $F T$ at 0.3 which is the value where the $F C$ can be activated. Hence, we need to 
find the PMS that will ensure that the $S O A C c^{B A T}$ will not drop below 0.2 and the lowest value of $S O A c c^{F T}$ is 0.3 . As in this case study the $S O A c c^{B A T}$ is more important than the $S O A c c^{F T}$ the first step will not be to take every possible combination of initial values for both $S O A c c^{B A T}$ and $S O A C c^{F T}$ but we will start from the target value of the previous case for the $S O A c c^{B A T}$, i.e. that in day 2 we must start at 0.241 . By repeating the same procedure as in the previous case but now for $S O A c c^{F T}$ we see that the minimum $S O A c c^{F T}$ that gets the system is 0.072 (row 1 of Table 4) and then using equation (8) the maximum MOES (row 3) for day 2. The required value is therefore derived from equation (9) which is 0.786 . Hence, in day 1 the ending values of $S O A c c^{B A T}$ and $S O A c c^{F T}$ must be 0.241 and 0.786 respectively. Using the given initial conditions for each accumulator (as stated in the previous section) the PGCC is calculated based on average weather data for day 1 and we see from Table 4 that it is not possible to have this combination using any PMS (Fig. 14). More specifically, while all PMSs give a value of $S O A C c^{B A T}$ higher than the required one (PMSs 1, 4 and 7 result closer to the target) there is no PMS that produces SOAcc ${ }^{F T}$ higher than 0.786 . The three PMSs that satisfy the condition for the $S O A c c^{B A T}$ produce a final value for $S O A c c^{F T}$ at $0.561,0.58$ and 0.561 respectively. Hence, a possible choice can be the $4^{\text {th }}$ PMS which produced the highest value of $\operatorname{SOAcc}{ }^{F T}(24)$.

Table 4: Complete results of case study 2

\begin{tabular}{|c|c|c|c|c|c|c|c|c|c|}
\hline & $\mathrm{PMS}_{1}$ & $\mathrm{PMS}_{2}$ & $\mathrm{PMS}_{3}$ & $\mathrm{PMS}_{4}$ & $\mathrm{PMS}_{5}$ & $\mathrm{PMS}_{6}$ & $\mathrm{PMS}_{7}$ & $\mathrm{PMS}_{8}$ & $\mathrm{PMS}_{9}$ \\
\hline $\operatorname{SOAcc}^{F T}\left(t_{\min }\right)$ & 0.082 & 0.072 & 0.072 & 0.082 & 0.072 & 0.072 & 0.082 & 0.072 & 0.072 \\
\hline $\operatorname{MOES}^{F T, q_{2}}(2)$ & 0.218 & 0.228 & 0.228 & 0.218 & 0.228 & 0.228 & 0.218 & 0.228 & 0.228 \\
\hline$S O A c c_{\min }^{F T, q_{2}}(1)$ & $\begin{array}{l}0.316 \\
0.335\end{array}$ & $\begin{array}{l}0.306, \\
0.335, \\
0.364, \\
0.393\end{array}$ & $\begin{array}{l}0.326, \\
0.355, \\
0.384, \\
0.413, \\
0.442, \\
0.471, \\
0.500, \\
0.529, \\
0.558\end{array}$ & $\begin{array}{l}0.3160 \\
0.3350\end{array}$ & $\begin{array}{l}0.306, \\
0.335, \\
0.364, \\
0.393\end{array}$ & $\begin{array}{l}0.326, \\
0.355, \\
0.384, \\
0.413, \\
0.442, \\
0.471, \\
0.500, \\
0.529\end{array}$ & $\begin{array}{l}0.316 \\
0.335\end{array}$ & $\begin{array}{l}0.306, \\
0.335, \\
0.364, \\
0.393\end{array}$ & $\begin{array}{l}0.326, \\
0.355, \\
0.384, \\
0.413, \\
0.442, \\
0.471, \\
0.500, \\
0.529, \\
0.558\end{array}$ \\
\hline $\max _{i \in Q}\left(\operatorname{SOAcc}_{\min }^{F T, q_{i}}(1)\right)$ & 0.335 & 0.393 & 0.5580 & 0.335 & 0.393 & 0.529 & 0.335 & 0.393 & 0.5580 \\
\hline$S O A c c_{\mathrm{req}}^{F T, q_{2}}(1)$ & 0.553 & 0.621 & 0.786 & 0.553 & 0.621 & 0.757 & 0.553 & 0.621 & 0.786 \\
\hline $\operatorname{SOAcc}_{T A R}^{F T}(2)$ & 0.786 & & & & & & & & \\
\hline
\end{tabular}




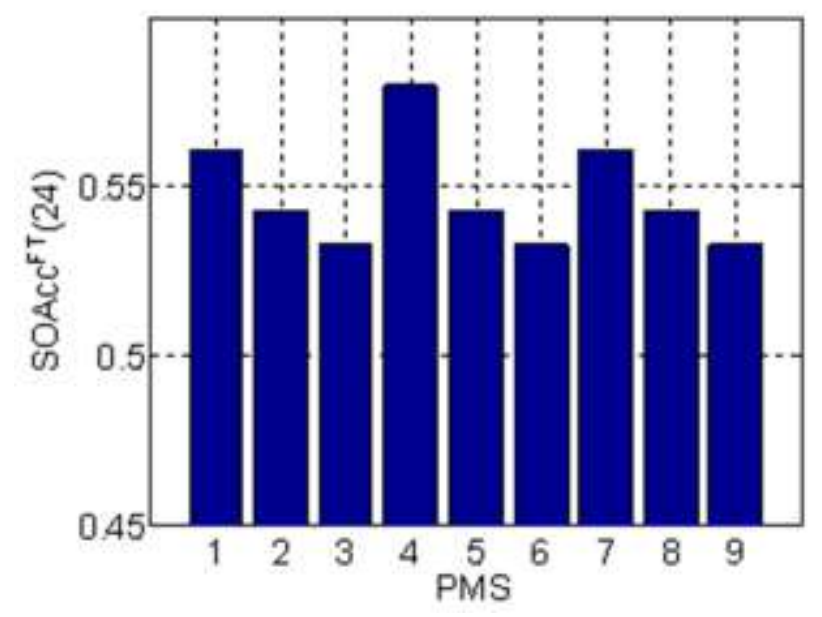

Figure 14: Last value of $S O A c c^{B A T}$ in day 1 for the 9 PMSs for case study 2.

\subsubsection{Adaptive operation}

Finally, in this subsection we compare the response of the system under study in the case where the proposed method is used against a case where a single PMS is employed for a whole year as it is usually done in similar systems. As a case study we use PMS1 and it is found that the DSL is operated for 443 hours while the $F C$ for 2397 hours. Also, at the end of the year the $F T$ is completely empty and the $F C$ in total produces $2.397 \mathrm{MWh}$. In the case of the adaptive method, various PMSs are chosen throughout the year (Fig. 15). The DSL is operated for 365 hours, i.e. there is an improvement of almost $18 \%$ compared to the constant use of PMS1. Furthermore, the FC delivers 2.365MWh in 1952 hours of operation hence it operates $19 \%$ less (i.e. it is protected more) producing almost the same amount of power as in PMS1. In order to see why the new adaptive method produces better results, we show in Fig. 16a the number of hours that the DSL is operated in each day, and the values of $S O A c c^{B A T}$ and $S O A c c^{F T}$ at end of each day for both cases. Notice that in the adaptive case the $S O A c c^{B A T}$ drops to the limit of 0.2 less frequently (Fig. 16b) while the charge-discharge cycles are considerably smoother supporting the prolongation of the battery life. Fig, 16c shows the profile of the stored hydrogen. For the adaptive case it indicates a more intense usage of the $F C$ (i.e. less hydrogen available in the $F T$ due to higher consumption) in 
the period between months 2 and 4 to avoid using the $D S L$. However, the $F C$ is overall utilized for fewer hours hence the adaptive case supports a better management of the system.

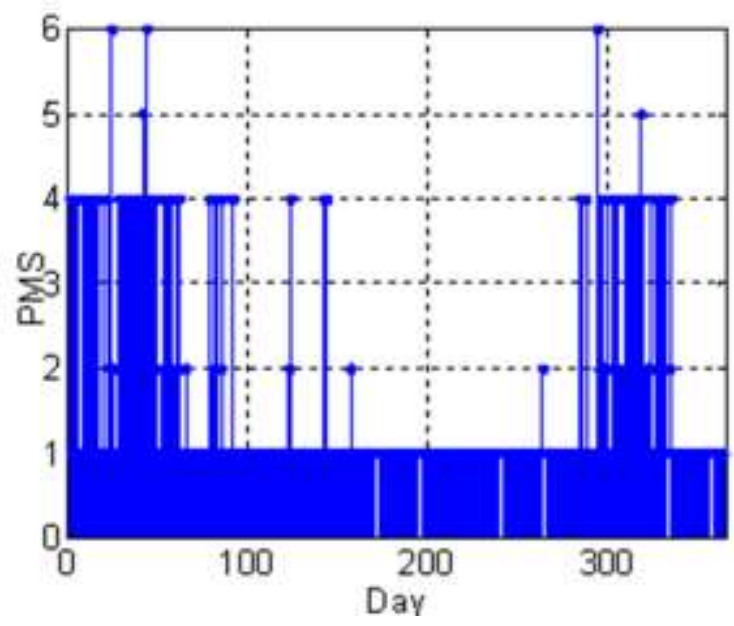

Figure 15: PMS used in each day for case study 3.
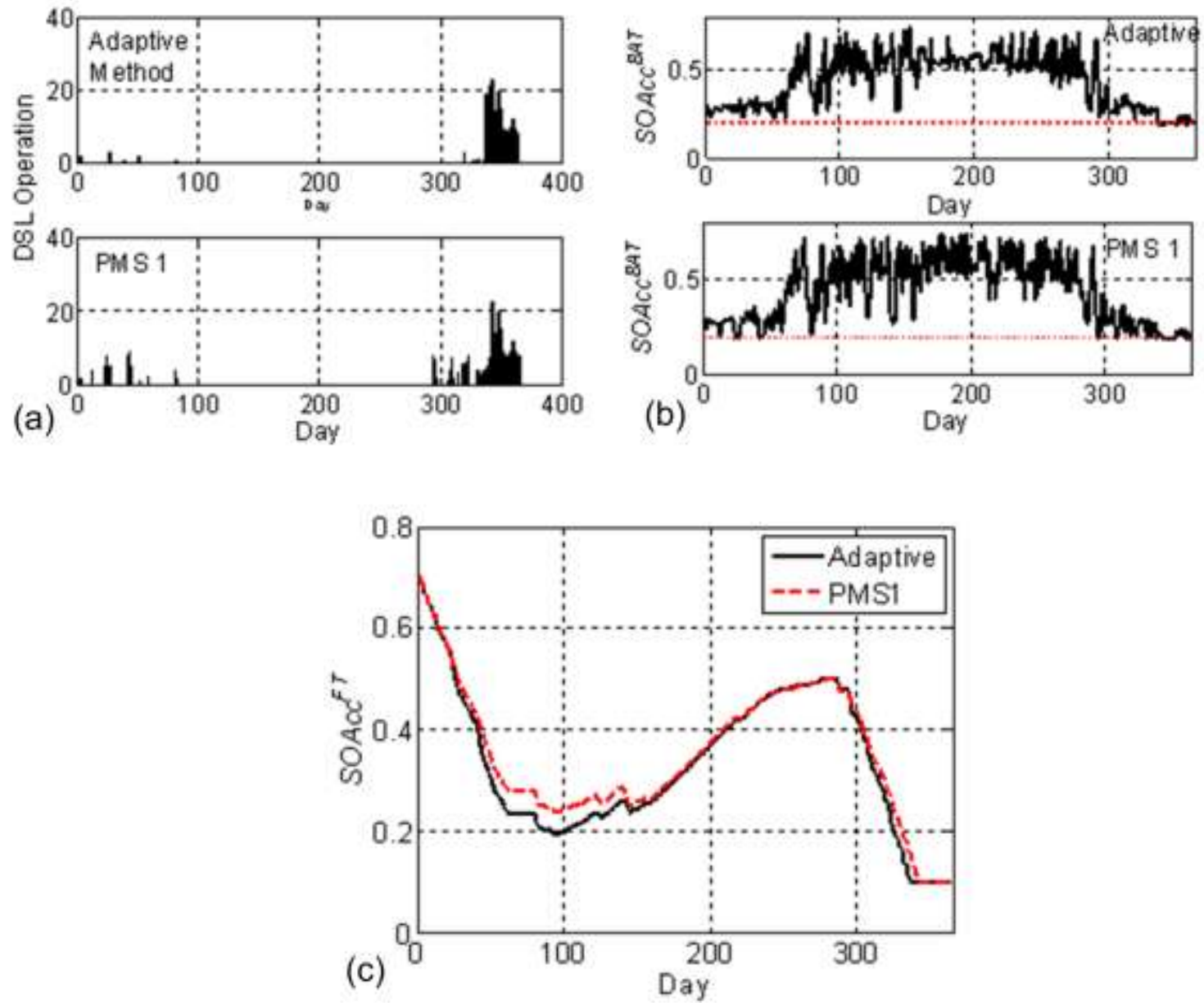

Figure 16: a) Operation of DSL, b) $S O A c c^{B A T}$ and c) $S O A c c^{F T}$ 
At this point it has to be mentioned that it is possible to use another PMS instead of 1 or another configuration of the system and to further minimise or even null the usage of the DSL. For example if PMS 9 is used (where the power of $F C$ equals the power deficit) then as long as there is available hydrogen in the $F T$, the $D S L$ will not be activated. However this will require an $F C$ of much larger capacity. In this case study for PMS 1 the $F C$ is rated at $1 \mathrm{~kW}$ while for PMS 9 the average power of the $F C$ is almost $3 \mathrm{~kW}$ with a peak value at $4 \mathrm{~kW}$. Hence, a much more expensive FC would be required in PMS 9. Similar issues appear in the other PMSs: for example in PMS 4 the DSL was operated only for 327 hours but it was activated 174 times versus 365 hours and 51 times using the proposed adaptive method. With the latter method the DSL operated 11\% more but was activated and deactivated $70 \%$ fewer times. Hence, as the frequent activations and deactivations of various devices can have a detrimental effect on their anticipated lifetime, the adaptive method proposed here prolongs their operation and facilitates their maintenance.

\section{Conclusions}

The presented work has addressed the implementation of the PGCC method for the analysis and adaptive operation of hybrid power generation smartgrids. Clearly, the use of PGCC analysis in such systems is not straight-forward. The interaction of sub-systems with different technical operating requirements imposes different limits and operating goals which often need to be considered simultaneously during the analysis. This work pointed out several such issues and proposed ways of incorporating them within the PGCC method both as graphical and mathematical representations. In particular, the graphical representations were supported by a generic mathematical framework which allows the automated implementation of the PGCC method for an extensive system operating horizon. This framework also facilitates the processing of short-term operating information within reduced time intervals, allowing efficient decisionmaking regarding the interactions of multiple sub-systems which result in efficient operation. The proposed PGCC analysis and the adaptive management method was built on a flexible model 
which is able to capture numerous potential interactions in the form of PMS by exploiting generic structural and temporal system features. The presented case studies exemplified different aspects of the proposed method, highlighting the benefits of combining the graphical analysis tools with the rigorous mathematical formulations.

\section{References}

[1] Giaouris D., Papadopoulos A. I., Ziogou C., Ipsakis D., Voutetakis S., Papadopoulou S., Seferlis P., Stergiopoulos F., Elmasides C. Performance investigation of a hybrid renewable power generation and storage system using systemic power management models. Energy 2013; 61: 621-35.

[2] Bandyopadhyay S. Design and optimization of isolated energy systems through pinch analysis. Asia-Pacific Journal of Chemical Engineering 2011; 6: 518-26.

[3] Wan Alwi SR, Rozali NEM, Manan ZA, Klemeš JJ. A process integration targeting method for hybrid power systems. Energy 2012; 44: 6-10.

[4] Smith R. Chemical Process: Design and Integration. New York: John Wiley and Sons; 2005.

[5] Linhoff B., Flower J.R. Synthesis of heat exchanger networks: I. Systematic generation of energy optimal networks. AIChE J. 1978; 24(4): 633-42

[6] Varbanov P.S., Fodor Z., Klemeš J.J. Total Site targeting with process specific minimum temperature difference ( $\Delta$ Tmin). Energy 2012; 44(1): 20-8

[7] Klemeš J.J. (ed). Process integration handbook. Cambridge: Woodhead Publishing; 2013.

[8] Rozali N.E.M, Wan Alwi S.R., Manan Z.A., Klemeš J.J., Hassan MY. Process integration techniques for optimal design of hybrid power systems. Applied Thermal Engineering 2013; 61: $26-35$.

[9] Wan Alwi S.R., Rozali N.E.M., Manan Z.A., Klemeš J.J. Design of hybrid power systems with energy losses. Chemical Engineering Transactions 2012; 29: 121-6. 
[10] Rozali N.E.M., Wan Alwi S.R., Manan Z.A., Klemeš J.J., Hassan M.Y. Process integration of hybrid power systems with energy losses considerations. Energy 2013; 55: 38-45.

[11] Rozali N.E.M., Wan Alwi S.R., Manan Z.A., Klemeš J.J., Hassan M.Y. Optimisation of Pumped-Hydro Storage System for Hybrid Power System Using Power Pinch Analysis. Chemical Engineering Transactions 2013, 35, 85-90.

[12] Rozali N.E.M., Wan Alwi S.R., Manan Z.A., Klemeš J.J., Hassan M.Y. Optimal sizing of hybrid power systems using power pinch analysis. Journal of Cleaner Production 2014; 71: 158-67.

[13] Wan Alwi, S. R. , Tin, O. S., Rozali, N. E. M., Manan, Z. A., Klemeš, J. J. New graphical tools for process changes via load shifting for hybrid power systems based on Power Pinch Analysis. Clean Technologies and Environmental Policy 2013; 15: 459-72.

[14] Ho W.S., Khor C.S., Hashim H., Macchietto S., Klemeš J.J. SAHPPA: a novel power pinch analysis approach for the design of off-grid hybrid energy systems. Clean Technologies and Environmental Policy 2013; 16(5): 957-70.

[15] Chen C.L., Lai C.T., Lee J.Y. A process integration technique for targeting and design of offgrid hybrid power networks. Chemical Engineering Transactions 2013; 35: 499-504.

[16] Chen C.L., Lai C.T., Lee J.Y. Transshipment model-based MILP (mixed-integer linear programming) formulation for targeting and design of hybrid power systems, Energy 2014: 65: 550-9.

[17] Ho W.S., Mohd Z.W.M.T., Haslenda H., Zarina A.M. Electric System Cascade Analysis (ESCA): Solar PV system. Electrical Power and Energy Systems 2014; 54: 481-486.

[18] Zahboune, H., Kadda, F.Z.; Zouggar, S.; Ziani, E.; Klemes, J.J. ; Varbanov, P.S. ; Zarhloule, Y., The new electricity system cascade analysis method for optimal sizing of an autonomous hybrid PV/wind energy system with battery storage, 5th International Renewable Energy Congress (IREC) 2014, 10.1109/IREC.2014.6826962. 
[19] Byeon G., Yoon T., Oh S., Jang G. Energy Management Strategy of the DC Distribution System in Buildings Using the EV Service Model. IEEE Transactions on Power Electronics $2013 ; 28(4): 1544-54$.

[20] Thanaa F.E., Mona N.E., Mohsen T.E. Energy flow and management of a hybrid wind/PV/fuel cell generation system. Energy Conversion and Management 2006; 47(9-10): 1264-80.

[21] Calderón M., Calderón A.J., Ramiro A., González J.F. Automatic management of energy flows of a stand-alone renewable energy supply with hydrogen support. International Journal of Hydrogen Energy 2010; 35(6): 2226-35.

[22] Rodatz P., Paganelli G., Sciarretta A., Guzzella L. Optimal power management of an experimental fuel cell/supercapacitor-powered hybrid vehicle. Control Engineering Practice 2005; 13(1): 41-53.

[23] Voutetakis S., Seferlis P., Stergiopoulos F., Papadopoulou S., Papadopoulos A.I., Ipsakis D., Ziogou C., Elmasides C. Design, Optimization and Control of Power Systems based on Renewable Energy Sources with Hydrogen Production, Storage and Utilization. New York: Nova Science Publishers Inc.; 2011

[24] Ipsakis D., Voutetakis S., Seferlis P., Stergiopoulos F., Elmasides C. Power management strategies for a stand-alone power system using renewable energy sources and hydrogen storage. International Journal of Hydrogen Energy 2009; 34 (16): 7081-95.

[25] Ziogou C., Ipsakis D., Elmasides C., Stergiopoulos F., Papadopoulou S., Seferlis P., Voutetakis S. Automation infrastructure and operation control strategy in a stand-alone power system based on renewable energy sources. Journal of Power Sources 2011; 196 (22):948899. 
[26] Moghaddam A.A., Seifi A., Niknam T., Pahlavani M.R.A. Multi-objective operation management of a renewable MG (micro-grid) with back-up micro-turbine/fuel cell/battery hybrid power source, Energy 2011; 36: 6490-507.

[27] Moradi M.H., Hajinazari M., Jamasb S., Paripour M. An energy management system (EMS) strategy for combined heat and power (CHP) systems based on a hybrid optimization method employing fuzzy programming. Energy 2013; 49: 86-101.

[28] Mehleri E.D., Sarimveis H., Markatos N.C., Papageorgiou L.G. A mathematical programming approach for optimal design of distributed energy systems at the neighbourhood level. Energy 2012; 44: 96-104.

[29] Buoro D., Casisi M., De Nardi A., Pinamonti P., Reini M. Multicriteria optimization of a distributed energy supply system for an industrial area, Energy 2013; 58: 128-37.

[30] Piacentino A., Barbaro C., Cardona F., Gallea R., Cardona E. A comprehensive tool for efficient design and operation of polygeneration-based energy $\mu$ grids serving a cluster of buildings. Part I: Description of the method, Applied Energy 2013; 111: 1204-21.

[31] Coroamă I., Chicco G., Gavrilaş M., Russo A. Distribution system optimisation with intraday network reconfiguration and demand reduction procurement. Electric Power Systems Research 2013; 98: 29-38.

[32] Chicco G., Mancarella P. Matrix modelling of small-scale trigeneration systems and application to operational optimization, Energy 2009; 34(3): 261-73.

[33] Kopanos G.M., Georgiadis M.C., Pistikopoulos E.N. Energy production planning of a network of micro combined heat and power generators. Applied Energy 2013; 102: 1522-34.

[34] Castañeda M., Cano A., Jurado F., Sánchez H., Fernández L.M. Sizing optimization, dynamic modeling and energy management strategies of a stand-alone PV/hydrogen/battery-based hybrid system. International Journal of Hydrogen Energy 2013; 38(10): 3830-45. 
[35] Rodatz P., Paganelli G., Sciarretta A., Guzzella L. Optimal power management of an experimental fuel cell/supercapacitor-powered hybrid vehicle. Control Engineering Practice 2005; 13(1): 41-53.

[36] Hooshmand A, Malki HA, Mohammadpour J. Power flow management of microgrid networks using model predictive control. Computers and Mathematics with Applications 2012; 64(5): 869-876.

[37] Sakhare A., Davari A., Feliachi A. Fuzzy logic control of fuel cell for stand-alone and grid connection. Journal of Power Sources 2004; 135(1-2): 165-76.

[38] Trifkovic M., Sheikhzadeh M., Nigim K., Daoutidis P. Modelling and control of a renewable energy system with hydrogen storage. IEEE Transactions on Control Systems Technology 2014; 22(1): 169-79.

[39] Lasseter R.H. Smart Distribution: Coupled Microgrids. Proceedings of the IEEE 2011; 99(6): 1074-82.

[40] Nehrir M.H., Wang C., Strunz K., Aki H., Ramakumar R., Bing J., Miao Z., Salameh Z. A Review of Hybrid Renewable/Alternative Energy Systems for Electric Power Generation: Configurations, Control, and Applications. IEEE Transactions on Sustainable Energy 2011; 2(4): 392-403.

[41] Logenthiran T., Srinivasan D., Khambadkone A.M., Aung H.N. Multiagent System for RealTime Operation of a Microgrid in Real-Time Digital Simulator. IEEE Transactions on Smart Grid 2012; 3(2): 925-33.

[42] Chen C., Duan S., Cai T., Liu B., Hu G. Smart energy management system for optimal microgrid economic operation. IET Renewable Power Generation 2011; 5(3): 258-67.

[43] Trifkovic M., Alex Marvin W., Daoutidis P., Sheikhzadeh M., Dynamic real-time optimization and control of a hybrid energy system, AICHE Journal 2014, 10.1002/aic.14458. 
[44] Ipsakis D., Voutetakis S., Seferlis P., Stergiopoulos F., Papadopoulou S., Elmasides C. The Effect of the Hysteresis Band on Power Management Strategies in a Stand-Alone Power System Energy. Energy 2008; 33: 1537-50.

[45] Wang Y.P., Smith R. Wastewater minimization with flowrate constraints. Chemical Engineering Research and Design 1995; 73(A8): 889-904.

[46] Hallale N., Moore I., Vauk D. Hydrogen optimization at minimal investment. Petroleum Technology Quarterly 2003; 2: 83-90. 